\begin{tabular}{|c|c|c|}
\hline \multicolumn{3}{|c|}{$\begin{array}{l}\text { Anno 13, numero } 1 \quad \text { Gennaio - Aprile } 2018 \\
\text { Poste Italiane - Spedizione in abbonamento postale - 70\% aut. DCB / Genova nr. } 569 \text { anno } 2005 \\
\text { TESTATA INDIPENDENTE CHE NON PERCEPISCE CONTRIBUTI PUBBLICI (legge 250/1990) }\end{array}$} \\
\hline & IN QUESTO NUMERO & $\begin{array}{l}\text { Risk Management Magazine } \\
\text { Anno } 13, \mathrm{n}^{\circ} 1 \text { Gennaio - Aprile } 2018\end{array}$ \\
\hline & & $\begin{array}{l}\text { Direttore Responsabile: } \\
\text { Maurizio Vallino }\end{array}$ \\
\hline \multicolumn{2}{|r|}{$\begin{array}{l}\text { ARTICOLI A CARATTERE DIVULGATIVO } \\
\text { (non sottoposti a referaggio) }\end{array}$} & $\begin{array}{l}\text { Condirettore } \\
\text { Corrado Meglio } \\
\text { Consiglio scientifico }\end{array}$ \\
\hline 3 & $\begin{array}{l}\text { AIFIRM risponde al Comitato di Basilea } \\
\text { sul documento «Revisions to the minimum } \\
\text { capital requirements for market risk» } \\
\text { Lavoro della Commissione Aifirm Market Risk } \\
\text { - coordinatori Marco Bianchetti e Umberto } \\
\text { Cherubini }\end{array}$ & $\begin{array}{l}\text { Giampaolo Gabbi (Direttore del Consiglio Scientifico), } \\
\text { Ruggero Bertelli, Paola Bongini, Anna Bottasso, } \\
\text { Marina Brogi, Ottavio Caligaris, Simona Cosma, Paola } \\
\text { Ferretti, Andrea Giacomelli, Adele Grassi, Pier } \\
\text { Giuseppe Giribone, Duccio Martelli, Laura Nieri, } \\
\text { Pasqualina Porretta, Anna Grazia Quaranta, Enzo } \\
\text { Scannella, Cristiana Schena, Giuseppe Torluccio. }\end{array}$ \\
\hline \multicolumn{2}{|r|}{$\begin{array}{l}\text { ARTICOLI A CARATTERE SCIENTIFICO } \\
\text { (sottoposti a referaggio) }\end{array}$} & $\begin{array}{l}\text { Comitato di redazione } \\
\text { Sara Brianza, Emanuele Diquattro, Fausto Galmarini, } \\
\text { Rossano Giuppa, Aldo Letizia, Paolo Palliola, Enzo } \\
\text { Rocca, Fabio Salis }\end{array}$ \\
\hline 8 & $\begin{array}{l}\text { Recovery Plan: punti di forza e di } \\
\text { debolezza } \\
\text { di Fabio Verachi }\end{array}$ & $\begin{array}{l}\text { Vignettista: Silvano Gaggero } \\
\text { Proprietà, Redazione e Segreteria: } \\
\text { Associazione Italiana Financial Industry Risk } \\
\text { Managers (AIFIRM), Via Sile 18, } 20139 \text { Milano }\end{array}$ \\
\hline 25 & 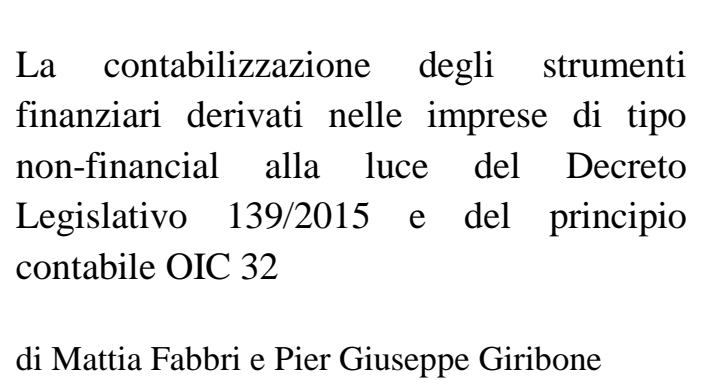 & $\begin{array}{l}\text { Registrazione del Tribunale di Milano } \mathrm{n}^{\circ} 629 \text { del } \\
\text { 10/9/2004 } \\
\text { ISSN 2283-7329 } \\
\text { E-mail: amministrazione@aifirm.it; } \\
\text { Tel. } 3896946315 \\
\text { Stampa: Algraphy S.n.c. - Passo Ponte Carrega 62-62r } \\
\text { 16141 Genova }\end{array}$ \\
\hline & & $\begin{array}{l}\text { Le opinioni espresse negli articoli impegnano } \\
\text { unicamente la responsabilità dei rispettivi autori } \\
\text { SPEDIZIONE IN ABBONAMENTO POSTALE AI } \\
\text { SOCI AIFIRM RESIDENTI IN ITALIA, IN } \\
\text { REGOLA CON L'ISCRIZIONE } \\
\text { Rivista andata in stampa il } 6 \text { Luglio } 2018\end{array}$ \\
\hline
\end{tabular}




\section{Processo di referaggio degli articoli proposti per la pubblicazione}

Gli articoli che sono proposti alla rivista per la pubblicazione sono sottoposti in forma anonima a due successivi livelli di referaggio.

Il primo livello di referaggio (di ammissibilità) viene effettuato sull'articolo dai membri del Consiglio Scientifico che ne valutano la congruità ai temi trattati dalla rivista.

Il secondo livello di referaggio (di pubblicabilità) viene effettuato sull'articolo da due referee scelti all'interno del Consiglio Scientifico o all'esterno tra accademici, ricercatori, esperti della materia, che ne valutano il contenuto e forma.

\section{Regolamento redazionale}

"Risk Management Magazine" è il periodico di AIFIRM (Associazione Italiana Financial Industry Risk Managers) ed interamente dedicato ai temi del risk management.

La sua struttura organizzativa prevede, oltre al direttore responsabile, un condirettore e un Consiglio Scientifico formato da accademici; quest'ultimo è garante della qualità e correttezza degli articoli pubblicati.

La rivista favorisce la diffusione di tutti i contenuti afferenti i temi del risk management, dagli aspetti normativi a quelli organizzativi e alle technicalities e vaglierà con interesse, per mezzo del Comitato Scientifico, i contributi che le perverranno.

Gli articoli proposti dovranno pervenire in formato Microsoft Word carattere Times New Roman 10 ed avere un numero di battute compreso tra 6.000 e 20.000; è gradita la presenza di tabelle e grafici.

La bibliografia deve essere redatta specificando accuratamente le fonti.

Si richiede la predisposizione di un Abstract in lingua inglese.

Le opinioni espresse negli articoli impegnano unicamente la responsabilità dei rispettivi autori.

Sul sito www.aifirm.it è pubblicata la Dichiarazione sull'etica e sulle pratiche scorrette nella pubblicazione dei lavori scientifici 
AIFIRM risponde al Comitato di Basilea sul documento «Revisions to the minimum capital requirements for market risk»

Lavoro della Commissione Aifirm Market Risk - coordinatori Marco Bianchetti (Intesa San Paolo) e Umberto Cherubini (Università di Bologna) ${ }^{1}$

\section{Abstract}

AIFIRM (Associazione Italiana Financial Industry Risk Managers), tramite la propria Commissione Rischi di Mercato, ha risposto alla consultazione proposta dal Comitato di Basilea sulla revisione dei requisiti minimi di capitale per il rischio di mercato.

Il documento "Revisions to the minimum capital requirements for market risk", posto in consultazione dal Comitato di Basilea dal 22 marzo al 20 giugno 2018, rappresenta un passaggio fondamentale nella ridefinizione delle regole internazionali volte a prevenire problemi analoghi a quelli verificatisi nel corso della crisi del 2007-2008 e, più in generale, a garantire una gestione più robusta dei rischi di mercato assunti dalle banche, rafforzandone la capacità di resistere a periodi di stress finanziario.

I commenti formulati da AIFIRM, sostanzialmente allineati con quelli formulati da altre realtà dell'industria finanziaria, si focalizzano sui temi ancora oggetto di affinamento, tra cui:

- la proposta di revisione degli scenari di correlazione fra i fattori di rischio e la modifica dei requisiti per il trattamento degli strumenti finanziari non lineari nell'ambito del nuovo metodo standardizzato, tipicamente utilizzato dalle banche piccole e medie,

- $\quad$ una gestione più efficiente del test di attribuzione del Profit\&Loss, che autorizza il metodo basato sui modelli interni tipicamente utilizzato dalle grandi banche.

Per quanto riguarda l'approccio semplificato proposto dal Comitato di Basilea, basato sulla ricalibrazione delle regole di Basilea II sul rischio di mercato, AIFIRM auspica la possibilità di applicazione di tale approccio ad un ampio insieme di banche che non presentano particolari livelli di rilevanza e complessità dell'attività di trading.

AIFIRM auspica che il confronto fra regolatori e industria finanziaria proceda, per arrivare a delle regole internazionali sulla gestione dei rischi efficaci nel proteggere sia le banche che gli investitori ed i risparmiatori dagli effetti negativi di nuove possibili crisi finanziarie.

Riferimenti: all'URL https://www.bis.org/bcbs/publ/d436.htm è possibile visionare il documento del Comitato di Basilea "Revisions to the minimum capital requirements for market risk" e tutti i commenti sottoposti dall'industria finanziaria.

\section{Testo del commento inviato da Aifirm}

\section{Introductory Remarks}

We welcome the opportunity to comment on the proposed revisions to the minimum capital requirements for market risk.

In our view, the consultative document issued by the BCBS takes significant steps to address the remaining challenges related to the implementation of the Fundamental Review of the Trading Book.

In general, we think that the extension of the implementation date and the new simplified alternative to the standardized approach represent important improvements to address outstanding issues. On the other hand, the extent of the proposed recalibration in risk weights seems to suggest a significant discrepancy between expected impacts and the data gathered so far. This could be a signal that further analysis is needed to identify a balanced and robust overall calibration, for both the standardized approach and the simplified alternative.

With reference to the internal model approach, we appreciate the effort undertaken to address outstanding issues in the context of profit\&loss attribution and non-modellable risk factors, but we think that further fine-tuning is required. In particular, in the context of statistical tests for profit\&loss attribution, we see the need of a monitoring period to determine an appropriate calibration of test thresholds.

\section{Specific comments}

\subsection{Standardised approach}

\section{Revisions to the treatment of liquid FX pairs}

We view as reasonable the proposal to treat currency pairs that arise as first order crosses of liquid currency pairs in the same way as liquid currency pairs. We notice, however, that the current proposal discretionally allows banks to apply the new treatment for crosses, thus softening the comparability of standardised capital requirements among banks. Therefore, given that the new treatment is fundamentally sound, we encourage the Committee to consider the possibility to make it mandatory.

${ }^{1}$ Documento sottoposto sia al Comitato Tecnico Scientifico di Aifirm (parere positivo di dieci accademici) sia al Consiglio di Aifirm (parere positivo di dodici professional), pervenuto il 7 giugno 2018 e approvato il 19 giugno 2018 


\section{Revisions to correlation scenarios}

Some risk factors are empirically observed to be consistently highly correlated in all market conditions. This might be the case, for example, of adjacent tenors on the same yield curve. We share the view that for these risk factors the "low correlations" scenario can produce correlations that are more conservative than what empirical data would support, and that this might make the outcome of the standardised approach overly conservative, or at least less meaningful than desired. The proposed revision to the "low correlations" scenario is based on a simple formula that, in our understanding, tends to reduce the difference between the "low correlations" scenario and the "medium correlations" scenario for correlation parameters higher than 0.75. In our view, the proposed change is a reasonable fix that improves, to some degree, the consistence of the "low correlations" scenario to what might happen in some stress scenarios.

\section{Revisions to capital requirements for non-linear instruments}

We notice that the revised framework for curvature risk remains strictly limited to instruments with optionality. This means that, for example, the curvature of a 30-years plain vanilla interest rate swap is not considered (see appendix 3.1 below). Moreover, in our understanding, the definition of linear instruments under the sensitivity-based method implies that instruments such as LIBOR-in-arrears Swaps or Constant Maturity Swaps are not subject to curvature and vega risk. While this can be a reasonable simplifying rule for most portfolios, it might make sense to include linear instruments alongside instruments with optionality in calculating curvature risk, and vega risk where applicable (e.g. LIBOR-in-arrears Swaps, Constant Maturity Swaps).

1. The approach to apply shock scenarios

We support the Committee's proposal to revise the approach such that consistent scenarios are applied to risk factors falling in the same "bucket" for credit spread, equity and commodity risk classes.

Regarding the alternative approach of defining "sectors" as a subset of each bucket and applying consistent scenarios at that level, we can identify the following merits and drawbacks.

\begin{tabular}{|l|l|}
\hline Merits & Drawbacks \\
\hline $\begin{array}{l}\text { Compared to the "consistent scenarios by bucket" } \\
\text { approach, the "consistent scenarios by sectors" might } \\
\text { lead to a more risk-sensitive approach and give results } \\
\text { that are less far from internal models. }\end{array}$ & $\begin{array}{l}\text { Defining "sectors" as a subset of each bucket would be a } \\
\text { significant change of the structure of the framework, and } \\
\text { would lead to a change in the data model for the FRTB } \\
\text { implementation in software systems. } \\
\text { Defining appropriate "sectors" would require a } \\
\text { significant effort to formulate a first consultative } \\
\text { proposal, collect and evaluate comments, carry-out a } \\
\text { quantitative impact assessment, etc. }\end{array}$ \\
\hline
\end{tabular}

Overall, we think that the drawbacks of the introduction of "sectors" overweight the merits, and we would there recommend the "bucket-based" approach.

2. Cliff effects caused by the approach used to calculate aggregate capital requirements

We agree with the finding that the formulae used to calculate the aggregate curvature risk capital requirement can cause cliff effects under certain conditions. In our understanding, these cliff effects arise from the fact that, within the aggregation formulae, only some terms are floored at zero. While we support the proposal to modify the formulae to avoid cliff effects, we are not sure that the proposed fix fully removes them. For example, consider the following formula:

$$
K_{b}^{-}=\sqrt{\max \left[0, \sum_{k} \max \left(C V R_{k}^{-}, 0\right)^{2}+\sum_{\mathbb{d} \neq k} \sum_{k} \rho_{k l} C V R_{k}^{-} C V R_{\mathbb{l}}^{-} \psi\left(C V R_{k}^{-}, C V R_{\mathbb{l}}^{-}\right)\right]}
$$

where $\psi\left(C V R_{\bar{k}}^{-}, C V R_{\bar{l}}^{-}\right)$is equal to 0 if both $C V R_{\bar{k}}^{-}$and $C V R_{\mathbb{R}}^{-}$are negative and equal to $1 \mathrm{in}$ all other cases. Assuming that we are aggregating two curvature terms with opposite signs and equal absolute value, i.e. $C V R_{1}^{-}=-C V R_{2}^{-}>0$, we have

$$
\begin{gathered}
\psi\left(C V R_{1}^{-}, C V R_{2}^{-}\right)=1_{x} \\
K_{b}^{-}=\sqrt{\max \left[0,\left(C V R_{1}^{-}\right)^{2}-2 \rho_{12}\left(C V R_{1}^{-}\right)^{2}\right]}=C V R_{1}^{-} \sqrt{\max \left(0,1-2 \rho_{12}\right)}
\end{gathered}
$$

Therefore, $K_{b}^{-}$as a function of the correlation parameter has the behaviour shown in the graph below, that might be considered a potential source of "cliff effects" for correlation values near 0.5 . 


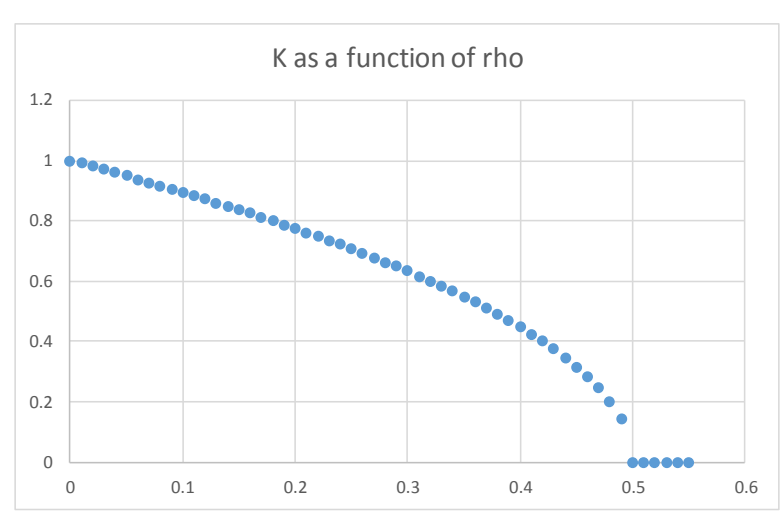

3. Potential double-counting of FX curvature risk

While we don't have empirical data to assess the materiality of the issue in real-world portfolios, we think that, even if the matter turned out to be of minor quantitative importance in most portfolios, it is a concrete issue, and encourage the Committee's effort to fix it. Given the cause of the issue, however, we are not sure that dividing by a pre-determined scalar X can result in an effective solution. Moreover, since the proposed scheme to fix the problem entails a discretion for banks, we notice that this could have drawbacks in terms of comparability of standardised capital requirements among banks. Therefore, if the Committee identifies a new treatment that it considers fundamentally sound, we encourage the Committee to consider the possibility to make it mandatory rather than discretionary.

\section{Revisions to risk weights}

We welcome the proposal to reduce the risk weights for some risk classes based upon its monitoring of the impact of the January 2016 standard. We notice that the current risk weights ranges shown in Annex A are still rather wide, particularly for equity spot prices and equity repo rates, and we suspect that further analysis is needed before the final recalibration. While we are unable to provide specific quantitative analysis in response to this document, we encourage the Committee not only to consider impact data provided by banks and feedback provided to this consultative document, but also to perform further empirical analysis before finalising the calibration.

One specific aspect that in our view should be included in the final revision is a recalibration of the risk weights for credit spread risk for covered bonds (Delta CSR non-securitisations). The current $4.0 \%$ risk weight for this bond bucket is the second highest among the eight investment grade bond buckets and does not seem to reflect the relative stability of covered bond spreads compared to other investment grade covered bonds. For example, the covered bonds risk weight is twice the corresponding risk weight for the technology and telecommunications bucket. We therefore suggest a recalibration, that should lead to a lower risk weight for covered bonds, taking into account at least the following aspects: (i) empirical evidence on historical volatility of covered bonds spreads, (ii) empirical evidence on the correlation of covered bond spreads with systematic risk, (iii) the significantly higher recovery rates on covered bonds, which should imply a lower sensitivity of their market value to changes in risk-neutral default probabilities of the issuer.

\subsection{Internal models approach}

\section{P\&L attribution test}

With reference to the input data for the P\&L attribution test, we welcome the fact that the committee recognised that differences between the HPL (Hypothetical P\&L) and the RTPL (Risk-theoretical P\&L) might arise from the use of different data providers. Removing these sources of discrepancy can, however, be costly and not trivial form an operational point of view.

In our view, this makes even more important to have a smoother mechanism to handle failures in the test. The introduction of the "traffic light" approach is an important update to smooth PLA test failure consequences for trading desks whose performance is not so poor with regards to front office pricing model. A consistent alternative to a binary "pass or fail" test design is very useful to increase the possibility that a bank can make use of a single model approach (Internal or Standard) while reducing complexity and effort of implementation. Trading desks in the "amber zone" could still make use of the internal model approach and retrieve data to calibrate and enhance their model. We note, however, that it retains some "digital" effects. In particular, we suggest that a trading desk that failed the test entering the "red zone", should be allowed to return in the "amber zone" when it meets the corresponding requirements.

Regarding the choice between the Kolmogorov-Smirnov and the Chi-squared test, we believe that a monitoring period will be necessary to determine an appropriate calibration based on real portfolios. One possible solution would be to start with a higher red zone threshold, that might be lowered at the end of the monitoring period.

Concerning Annex B, in which two alternatives are given for the definition of buckets (B.2 - Revisions to risk factor modellability), we support alternative 1, i.e. "banks must define the buckets they will use" (subject to one-to-one correspondence between buckets and risk factors used for RTPL), over alternative 2 which requires the use, at a minimum, of a set of standard buckets. As a further option we encourage the committee to consider a variant of alternative 2 with less granularity, especially for implied volatilities. 
With reference to Annex C, we support the provision stating that, subject to regulatory approval, a bank may assign individual traders to up to two trading desks.

\section{Non-modellable risk factors}

We welcome the fact that the Committee is open to the revision of the treatment of idiosyncratic equity risk in the context of non-modellable risk factors, allowing the recognition of diversification benefits. A revision of this aspect would address a current shortcoming of the framework. While we are not able to provide quantitative evidence, in our view the issue is very likely to be material at least for some banks, and the amendment proposed in the text of paragraph 190 as included in Box 3 in the consultative document would be an important improvement.

\subsection{Scope of market risk capital requirements}

\section{Treatment of structural FX positions}

Regarding investments in consolidated subsidiaries and non-consolidated affiliates, we welcome the proposal to allow the amount of structural FX positions that may be exempted from market risk capital requirements to be measured based on FX risk stemming from an investment, rather than from the amount of the investment itself. We also welcome the clarification that structural FX positions in foreign branches of a bank can be included in the scope of the structural FX exemption.

With reference to Annex E.1, we highlight that:

- $\quad$ it should be possible to hedge capital ratios both with derivatives and with cash instruments;

- all FX positions should be considered in the net and structural FX position, whatever its accounting classification, irrespective of the fact that it belongs to the banking book or the trading book, and the entity within the group by which it is booked;

- $\quad$ in general, hedging the capital ratios from FX risk will aim at reducing, rather than eliminating, the sensitivity of the ratios to exchange rates.

\section{Boundary between the trading book and the banking book}

With reference to equity investments in funds, we notice that the consultation paper seems to imply the exclusion from the trading book of funds, including hedge funds, for which no daily real prices are available. While we understand that there might be concerns with the inclusion in the trading book of illiquid funds for which pricing information is available only on a non-daily basis, we notice that moving these instruments to the banking book might be, in many cases, inconsistent with the way they are traded and risk managed. An alternative solution might be to allow non-look-through funds in the trading book with an additional capital charge, or - if this is not feasible - to allow trading book classification for funds that meet at least one of the following conditions: (i) daily prices or (ii) daily look through.

\subsection{Simplified alternative to the standardised approach}

In line with the comments we provided on the "Simplified alternative to the standardised approach to market risk capital requirements" consultative document, we welcome the proposal to allow a fairly wide range of banks to adopt a simplified approach based on the Basel II market risk framework.

Given that the adoption of the simplified alternative is subject to supervisory approval, we see a risk that different approaches taken by national supervisors can create disparities.

We therefore suggest that:

- the indicative criteria could be supplemented by a few additional criteria, for example:

○ the bank should not use an internal model for credit risk (as an indirect indicator of complexity);

o trading activities should not represent the main revenue source for the bank.

- if a bank satisfies all the indicative eligibility criteria (i.e. the three criteria listed in the document and any additional criteria), the national supervisor should either allow the use of the simplified alternative or explain the specific reasons that prevent its use.

While the simplified approach should not be available to G-SIBs, it might make sense to allow subsidiaries and branches of GSIBs with limited and unsophisticated trading activities to apply it. In this case, risk weighted assets calculated at the entity level using the simplified approach should be considered when calculating consolidated risk weighted assets at the group level.

For what concerns the capital requirements, we notice that an ample prudential margin seems to be embedded in the proposed scaling factor ranges.

While we understand that the recalibration proposed is intended to be comparable with, but slightly more conservative than, the revised "full" standardised approach, we are concerned that the multiplier range for general and specific equity risk (3.003.50) might be too high in comparison to the ranges for other risk classes (1.25-2.50). The large difference in multipliers seems to imply that the Basel II standardised approach strongly underweights general and specific equity risk, but we are not aware of empirical evidence supporting this conclusion. 


\section{Appendices}

\subsection{Curvature of IRS}

According to the standard market practice, plain vanilla Interest Rate Swap (IRS) is typically priced using the so-called "multi-curve approach", where an index (or forward) yield curve (e.g. Euribor 6M yield curve) is used to calculate the floating cash flows and another discounting yield curve is used to discount future cash flows at the valuation date (e.g. EUR OIS yield curve). Hence the IRS shows a linear dependence with respect to the forward yield curve (since cash flows depends linearly on forward rates), such that only its delta sensitivity is non-null. On the other hand, the IRS shows a marginal non-linear dependence with respect to the discounting curve (since discount factors depends non-linearly on discount rates), leading to a residual non-null curvature. Such residual curvature is small but observable in long maturity IRS, as shown in the table below for a $30 Y, 100 \mathrm{~mm}$ notional IRS, where the gamma $1 \mathrm{bp}$ is 683 EUR.

\begin{tabular}{|c|c|c|}
\hline Leg 1 & Leg 2 & Valuation results \\
\hline Receive & Pay & NPV: $0.00 \mathrm{EUR}$ \\
\hline Notional: EUR 100 MM & Notional: EUR 100 MM & DV01: 240,484.13 EUR/1bp \\
\hline Maturity: $30 \mathrm{Y}$ & Maturity: 30Y & Gamma: $683.07 \mathrm{EUR} / 1 \mathrm{bp}{ }^{2}$ \\
\hline Fixed rate: $1.57267 \%$ & Floating Rate: Euribor $6 \mathrm{~m}$ & \\
\hline Conventions: Annual, $30 / 360$ & Conventions: Semi Annual, ACT/360 & \\
\hline
\end{tabular}

We notice that such gamma sensitivity may be comparable to the gamma sensitivity of a similar non-linear instrument, such as the Swaption shown in the table below (Swaption receiver 1Yx5Y strike close to ATM 100mm notional amount, gamma 1bp 507 EUR).

\begin{tabular}{|c|c|c|}
\hline European Swaption & & Valuation results \\
\hline Position: short receiver & Strike: $0.68 \%$ & NPV: $-726,320.48 \mathrm{EUR}$ \\
\hline Notional: EUR 100 MM & ATM Strike: 0.702095\% & DV01: $-23,811.72 \mathrm{EUR} / 1 \mathrm{bp}$ \\
\hline Expiry: 1Y & $\begin{array}{c}\text { Underlying Swap: pay fixed } \\
\text { (Annual, 30/360), receive Euribor } \\
\text { 6m (Semi Annual, ACT/360) }\end{array}$ & Gamma: $-507.21 \mathrm{EUR} / 1 \mathrm{bp}{ }^{2}$ \\
\cline { 3 - 3 } Tenor: 5Y & Vega: $-19,574.52 \mathrm{EUR} / 1 \mathrm{bp}$ \\
\hline
\end{tabular}




\section{Recovery Plan: punti di forza e di debolezza}

di Fabio Verachi (Intesa San Paolo) ${ }^{1}$

\section{Abstract}

Recovery and resolution planning is a key element of the regulatory response agreed by G20 leaders to the problem of 'toobig-to-fail' banks and financial institutions. The issue emerged as a result of the disorderly failure of Lehman Brothers and the related consequences on the financial system at a global level. As per decision of the G20, the European Commission, following a long consultation process, adopted a Directive establishing a recovery and resolution framework for credit institutions and investment firms in June 2014 (Directive 2014/59/EU, also known as Bank Recovery and Resolution Directive or BRRD). In Italy, the BRRD transposition law entered into force in January 2016.

Among this context, the Directive requires banking institutions to draw-up recovery plans. The recovery plan must describe the measures which each institution intends to adopt to restore its own financial position in the event of a significant deterioration. These measures should not envisage any use of public resources and must include suitable conditions and procedures for ensuring a swift recovery. This process must be updated annually or any time there is a substantial change to the legal or organizational structure, or in the activities or financial position of the institution.

A specific AIFIRM Commission has been charged to analyze these issues and in particular the strengths and weaknesses of recovery plans in the Italian banking system. The resulting position paper is focused on reverse stress scenarios and listing of recovery actions to be reported in the plan, as well as the need to ensure consistency with the Risk Appetite Framework, the strategic guidelines and the ICAAP process. The analysis has also confirmed the necessity of considering all relevant risks also incorporating prospective assessments, an appropriate methodological framework known and shared by the internal structures, an adequate and formalized documentation system, an integration of management activities specifying roles and responsibilities of different internal functions.

Additionally, in the light of the recent IFRS 9 introduction, the AIFIRM Commission does not emphasize any disrupt changing in the underlying logics even if it certainly has an impact on the way of creating scenarios, on prospective indicators, on recovery actions and on governance mechanisms. Nevertheless, the IFRS 9 principle represents an opportunity to favor the transition from a static to a dynamic management vision, in which planning and budgeting activities should be continuously supported by the predictive risk management processes.

Finally, the position paper contains the results of a survey carried out on Italian Banks, highlighting on the one hand a useful operating contribution in identifying and making the appropriate short-term corrections to the respective recovery plans and, on the other hand, a reference framework to guide the future evolutions referring to the experiences observed in best practices and the interlocution with the Supervisory Authorities.

\section{Introduzione}

La redazione dei piani di risanamento e di risoluzione è parte integrante della risposta regolamentare concordata dai capi di Stato del G20 al problema della risoluzione transfrontaliera delle banche e delle istituzioni finanziarie too-big-to-fail, resosi evidente dopo il fallimento disordinato di Lehman Brothers e le conseguenze sul sistema finanziario a livello globale.

Su indicazione del G20, il Financial Stability Board (FSB) ha redatto i Key Attributes of Effective Resolution Regimes for Financial Institutions, documento pubblicato nell'ottobre 2011, che definisce le caratteristiche e gli elementi necessari per l'implementazione a livello nazionale/regionale di quadri normativi ed efficaci regimi di risoluzione. I Key Attributes, che necessitano di implementazione da parte delle autorità nazionali e regionali competenti, sono indirizzati in primis alle cosiddette istituzioni finanziarie di rilevanza sistemica a livello globale (G-SIFIs) e ad altre imprese il cui fallimento avrebbe un impatto non trascurabile sulla stabilità finanziaria.

Tra gli strumenti individuati dal FSB spicca la necessità di redigere piani di risanamento e di risoluzione, elementi fondamentali della strategia di prevenzione e risoluzione delle crisi. In particolare, i piani di risanamento (Recovery Plan) stabiliscono le modalità e le misure con cui intervenire in fase precoce per ripristinare la sostenibilità economica a lungo termine di un'istituzione in caso di grave deterioramento della situazione finanziaria.

Al fine di porre in essere le decisioni prese a livello internazionale dal G20, la Commissione Europea ha presentato la proposta di Direttiva che istituisce un quadro di risanamento e di risoluzione delle crisi degli enti creditizi e delle imprese di investimento (Bank Recovery and Resolution Directive, BRRD). La nuova normativa è stata quindi recepita a livello nazionale nel Testo Unico Bancario (Decreto legislativo 16 novembre 2015, n. 180 - di trasposizione della BRRD - e Decreto legislativo 15 novembre n. 181 - di modifica del Testo Unico Bancario).

La legge italiana è entrata in vigore il $1^{\circ}$ gennaio 2016, fatta eccezione per la sezione che introduce $1^{\prime}$ extended depositor preference, che entrerà in vigore a partire dal gennaio 2019. Le disposizioni prevedono l'applicazione del bail-in che introduce la subordinazione di tutte le obbligazioni senior unsecured rispetto a tutte le tipologie di depositi: non solo retail, SME e covered deposit (con depositi uguali o inferiori a $€ 100.000$ ), come previsto dalla BRRD, ma anche ai depositi superiori a $€ 100.000$, quali depositi interBancari e corporate.

Come recentemente avvenuto nel caso di alcune banche anche di dimensioni significative, gli aiuti di Stato non sono completamente esclusi dalla normativa, ma devono essere limitati al minimo necessario e subordinati ad una adeguata

${ }^{1}$ Le opinioni espresse nella presentazione rappresentano esclusivamente il punto di vista dell'autore e non riflettono necessariamente quello di Intesa Sanpaolo. L'articolo è stato inoltre sottoposto a doppio referaggio anonimo, pervenuto in data 20/06/2018 e accettato il 27/06/2018. 
condivisione degli oneri da parte degli investitori (cd. burden sharing). La BRRD attribuisce importanti poteri alle autorità competenti e alle autorità di risoluzione in tre ambiti:

i) misure preparatorie e stesura dei piani di risanamento e risoluzione per ridurre al minimo i rischi di problemi potenziali (poteri di preparazione e prevenzione);

ii) in caso di problemi incipienti, la capacità di arrestare il deterioramento della posizione della Banca in fase precoce in modo da evitarne l'insolvenza (poteri di intervento precoce);

iii) se l'insolvenza di un ente suscita preoccupazioni di interesse pubblico (ai sensi dell'art.32 della Direttiva), l'utilizzo di strumenti per risanare o liquidare la Banca in modo ordinato, preservandone le funzioni essenziali e limitando al massimo l'esposizione dei contribuenti alle perdite in caso di insolvenza (poteri di risoluzione).

L'applicazione della BRRD si inserisce inoltre nel contesto normativo sorto in risposta alla crisi dei Paesi dell'Eurozona e culminato nella creazione dell'Unione Bancaria. Tale progetto, composto da tre pilastri normativi ${ }^{2}$, ha lo scopo di spezzare il circolo vizioso che unisce banche e Stati sovrani di appartenenza, di consentire una corretta trasmissione delle politiche monetarie all'economia reale, di estendere la portata dell'Unione economica e monetaria garantendo la stabilità finanziaria dell'Eurozona e di ristabilire la fiducia nel settore finanziario.

In linea con queste indicazioni normative, il presente lavoro si propone l'obiettivo di illustrare lo stato dell'arte del sistema bancario italiano in merito alla predisposizione dei piani di recovery e di cercare di definire un percorso di avvicinamento alle migliori prassi internazionali, anche in funzione delle principali tendenze evolutive da integrare nella governance degli intermediari finanziari. In ossequio a questi principi, sono state effettuate diverse riflessioni che si propongono lo scopo di:

o proporre una chiave di lettura utile ad individuare i punti di forza e di vulnerabilità delle banche;

o facilitare la comprensione delle dinamiche sottostanti alla capacità delle banche di rispondere alle situazioni di stress attraverso l'identificazione di opportune azioni di recovery;

o illustrare le innumerevoli opzionalità delle azioni e la loro efficacia;

o fornire un quadro sintetico, anche attraverso un'apposita survey ${ }^{3}$ distribuita tra un panel di banche attraverso $\mathrm{i}$ membri AIFIRM (Figura 1), sullo stato dell'implementazione as is del Recovery Plan nel sistema Bancario italiano, presentando i principali approcci adottati a livello nazionale.

\section{Figura 1. Composizione del panel di partecipanti alla survey}

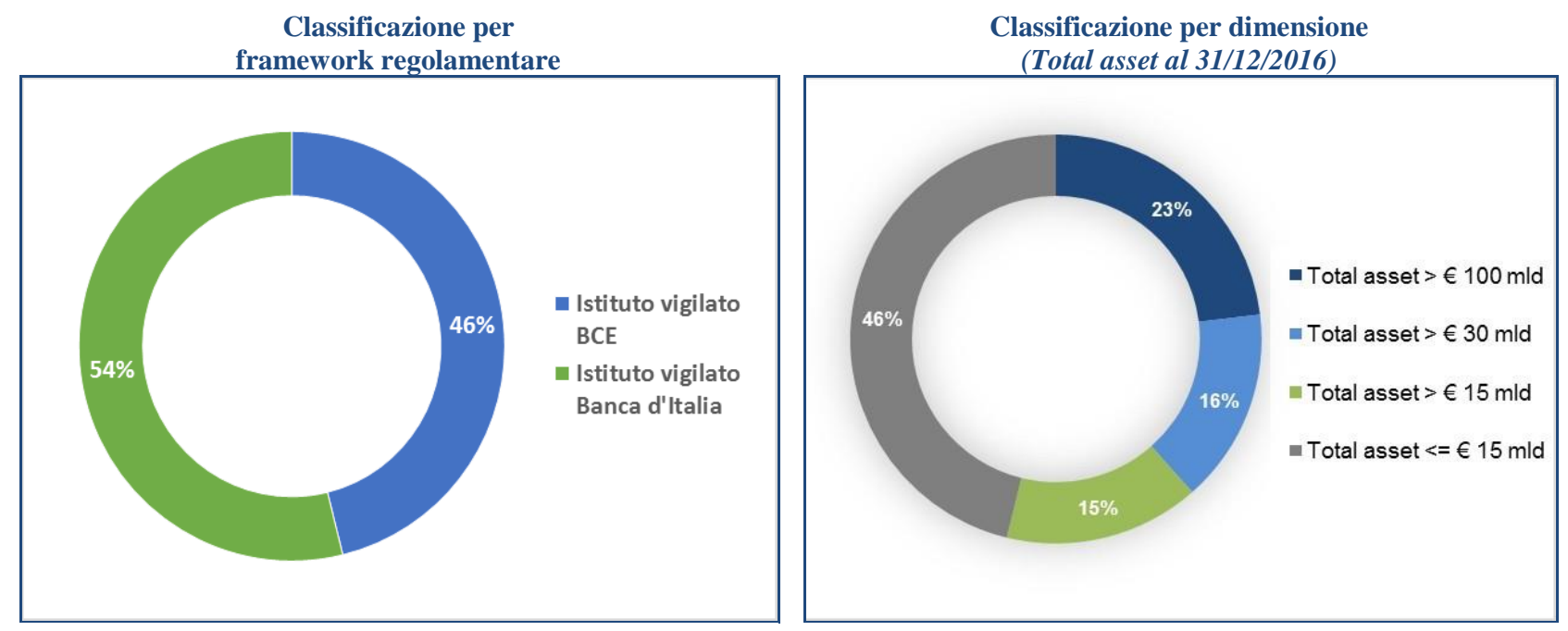

\footnotetext{
${ }^{2}$ Il primo pilastro dell'Unione Bancaria è rappresentato dal meccanismo di vigilanza unico (Single Supervisory Mechanism, SSM) che conferisce alla BCE poteri di vigilanza sulle banche dei Paesi aderenti. L'accentramento delle funzioni di vigilanza assicura una supervisione integrata a livello europeo e consente un maggiore level playing field attraverso l'applicazione di regole comuni, derivanti principalmente dalle norme in tema di requisiti prudenziali (Regolamento e Direttiva sui Requisiti Patrimoniali, i.e. CRR/CRD IV).

Il secondo pilastro dell'Unione Bancaria è volto ad assicurare maggiore coordinamento nella gestione delle crisi bancarie attraverso la creazione di un meccanismo unico di risoluzione. Tale meccanismo è composto da un'autorità di risoluzione centrale (Single Resolution Board, SRB) e da un fondo unico di risoluzione (Single Resolution Fund, SRF), costituito con i contributi del settore finanziario.

Il terzo pilastro dell'Unione Bancaria, ancora in discussione, dovrebbe prevedere la creazione di un fondo europeo a garanzia dei depositi, allo scopo di assicurare uniformità nella protezione dei correntisti a livello europeo e di evitare, in caso di crisi, corse allo sportello e trasferimenti di depositi in banche di Paesi UE percepiti come più solidi.

${ }^{3}$ Le analisi di dettaglio sui risultati della survey sono contenute in apposita sezione del Position Paper AIFIRM n. 11 "Recovery Plan: punti di forza e di debolezza" - giugno 2018.
} 


\section{Principali contenuti del Piano}

Nell'attuale contesto normativo di riferimento, l'Autorità di Vigilanza chiede annualmente alle banche di fornire un Piano di recovery approvato dagli organi societari. La redazione di tale documento comporta il coinvolgimento di una pluralità di strutture e funzioni aziendali riconducibili alle aree di governo che nei grandi gruppi si identificano con le aree Chief Financial Officer (CFO), Chief Risk Officer (CRO) e Chief Operating Officer (COO).

La predisposizione del Piano di recovery è quindi un processo articolato e complesso che prevede l'iterazione tra numerose funzioni interne il cui coordinamento può essere svolto da una struttura responsabile dell'intero processo operativo. L'impostazione deve essere parte integrante della governance e basarsi su:

- idonei sistemi aziendali di individuazione delle situazioni di crisi e delle azioni che devono essere intraprese in caso di attivazione;

- $\quad$ adeguati meccanismi di governo e definizione delle responsabilità a livello organizzativo.

La struttura del documento del Piano (di seguito recovery book) può essere articolata nelle seguenti macro sezioni.

\section{Sezione 1 - Quadro Normativo:}

- Breve rappresentazione del quadro normativo in tema di recovery.

\section{Sezione 2 - Struttura dell'intermediario:}

- Visione di sintesi dei piani strategici, descrizione della Banca e della governance del Piano per le entità incluse nel perimetro.

\section{Sezione 3 - Piano di recovery:}

- Definizione, approvazione e processo di aggiornamento del Piano di recovery: descrizione del modello di governance, delle macro fasi di definizione e approvazione del Piano, degli attori coinvolti, dei principali momenti di condivisione interna e del relativo processo di aggiornamento.

- Il processo decisionale interno per l'attivazione del Piano di recovery: definizione del processo di escalation, del ruolo e coinvolgimento di top management e CdA, nonché delle modalità di interazione con l'autorità di Vigilanza.

- Risk Appetite Framework (RAF) e indicatori di recovery: definizione dei trigger per l'attivazione del Piano e identificazione di indicatori e strumenti di early warning in coerenza con le raccomandazioni dell'EBA e con le impostazioni del modello complessivo di Risk Appetite della Banca.

- Scenari di Stress: definizione di scenari di stress (idiosincratici/sistemici e ad impatto graduale/rapido e loro combinazioni) e valutazione dei relativi impatti in termini di adeguatezza patrimoniale e liquidità.

- Azioni di recovery: definizione delle azioni di recovery con relativa descrizione degli impatti (finanziari, operativi, ecc.) e analisi di fattibilità, ostacoli, implicazioni interne/esterne, processo decisionale e piano di implementazione.

- Gestione della continuità delle operazioni: focus sulle azioni di recovery per individuare le eventuali azioni necessarie a garantire la continuità operativa delle funzioni e degli shared services critici per la Banca.

- Strategie di comunicazione: definizione delle linee guida e piano di comunicazione interna ed esterna per ogni specifica azione di recovery e descrizione delle politiche di gestione delle informazioni in situazioni di stress.

- Gestione delle informazioni: overview dell'architettura tecnologica e applicativa dell'ente e identificazione delle fonti informative utilizzate per la definizione del Piano.

\section{Sezione 4 - Follow-up:}

- Individuazione delle modalità di gestione dei possibili futuri cambiamenti del Piano e delle 'misure preparatorie' volte a garantire l'effettiva percorribilità delle azioni di risanamento, superando gli eventuali impedimenti all'efficace attuazione delle stesse.

La struttura e l'articolazione di un recovery book, i ruoli e le responsabilità che fanno capo alle funzioni aziendali per l'alimentazione del documento, nonché il processo di aggiornamento dello stesso può essere illustrata come di seguito (Figura 2). 
Figura 2. Struttura e articolazione di un recovery book, ruoli e responsabilità che fanno capo alle funzioni aziendali per l'alimentazione del documento e processo di aggiornamento dello stesso

Struttura Recovery Plan
Elenco capitoli

\section{Contenuto componenti principali}

Analisi del gruppo

- Descrizione del Gruppo e delle interconnessioni interne

- Focus sulle L.E. incluse nel perimetro di analisi

- Analisi delle Funzioni Economiche a carattere sistemico svolte dal Gruppo e delle funzioni di supporto critiche al loro funzionamento (Shared Services)

Aggiornamento e attivazione Piano; RAF e trigger

- Descrizione del processo di aggiornamento del Piano

- Modello di Crisis Management e aspetti governance

- Descrizione degli indicatori di recovery individuati (trigger) e della loro coerenza con le policy esistenti (Risk Appetite Framework e Contingency Liquidity Plan)

Scenari di stress

- Descrizione della catena di eventi che porta da business as usual a situazione di stress con eventuale superamento dei recovery trigger

Azioni di recovery e gestione continuità

- Presentazione delle azioni di recovery a disposizione del Gruppo raggruppate secondo le seguenti categorie:

- Riduzione attivi di bilancio e cessione crediti

- Cessione partecipazioni di minoranza

- Cessione partecipazioni di controllo

- Azioni sul patrimonio

- Generazione Straordinaria di liquidità

- Valutazione degli impatti e gestione della continuità operativa

- Gestione della comunicazione interna ed esterna in recovery

Mappatura dati

- Descrizione dei processi di gestione delle informazioni necessarie per la stesura e l'analisi del recovery plan

Fonte: Elaborazioni da RTS on the content of recovery plans, EBA - 4 febbraio 2016.

\section{Governance del Piano di Recovery}

I ruoli e le responsabilità nell'ambito del processo di redazione del Recovery Plan risultano definiti sulla base della combinazione dei requirement normativi e del modello di business e di governance specifici della singola Banca. La normativa di riferimento sottolinea infatti la necessità per le banche di mettere in atto misure idonee per colmare il gap tra $\mathrm{i}$ rischi assunti nell'operatività corrente e le misure volte a ripristinare la situazione finanziaria a seguito di un deterioramento significativo della stessa. In questo contesto, la presenza di un'adeguata struttura di coordinamento dedicata, in grado di coinvolgere adeguatamente gli organi aziendali, i principali comitati e le diverse funzioni aziendali interessate dalle diverse fasi del processo, risulta essere di fondamentale importanza al fine di garantire che la consapevolezza dei rischi e delle azioni di recupero siano adeguatamente diffuse in tutta l'organizzazione.

La struttura di coordinamento (di seguito anche Capital Management e Recovery Plan ${ }^{4}$ ) deve garantire l'attivazione delle diverse funzioni aziendali coinvolte, dei referenti delle principali unità organizzative che rientrano nel perimetro di analisi, nonché delle strutture di riferimento delle diverse analisi da effettuare. Tale struttura potrebbe anche assumere la qualifica di 'unità di emergenza' in quanto, assicurando il coordinamento trasversale tra i diversi livelli ed unità organizzative coinvolte, garantisce l'esecuzione delle contromisure volte a mitigare tempestivamente i rischi dell'istituto.

In termini di best practice, di seguito si fornisce una sintesi delle principali attribuzioni riguardanti il processo di predisposizione del Piano di risanamento:

- la funzione di Gestione dei Rischi (di seguito anche Risk Management) provvede alla costruzione degli scenari di reverse stress test, identifica gli indicatori del Piano di risanamento e ne definisce le relative soglie, garantendone nel tempo l'adeguatezza, l'efficacia e la coerenza con il Risk Appetite Framework. Inoltre, di concerto con le Funzioni dell'Area CFO (Chief Financial Officer) può curare, in assenza di una struttura organizzativa dedicata, il coordinamento complessivo del processo di generazione del Piano ed essere coinvolta nel monitoraggio degli indicatori al fine di valutare la situazione economico-finanziaria dell'istituto creditizio al fine di prevenirne lo stato di crisi;

- le funzioni dell'area CFO (in particolare la struttura Pianificazione e Controllo di Gestione) svolgono le attività di analisi volte a valutare gli impatti interni, di natura operativa e finanziaria, ed esterni sulle funzioni critiche (cd. critical function) ed identificano le opzioni di recovery provvedendo a valutare, con il supporto delle altre funzioni coinvolte, la fattibilità, l'efficacia e la coerenza con il RAF e con la pianificazione strategica ed operativa. Inoltre, di

${ }^{4}$ Tale struttura può ovviamente assumere diverse denominazioni ed essere collocata nel CRO, nel CFO o nel COO. 
concerto con la Funzione Risk Management, la struttura Pianificazione e Controllo di Gestione può fornire supporto nella predisposizione del reporting informativo e proporre la formalizzazione/gestione dei meccanismi di escalation;

- le funzioni dell'area COO (in particolare la struttura Organizzazione) definiscono il processo e la governance di predisposizione e aggiornamento del Piano di risanamento nonché le fasi, i ruoli e le responsabilità degli attori coinvolti nel processo di attivazione, esecuzione e chiusura dello stato di recovery. Inoltre, tali strutture possono essere coinvolte nel fornire supporto sia nella predisposizione della business analysis al fine di individuare i critical shared service sia nella verifica degli impatti interni di natura operativa delle azioni di recovery;

- le funzioni di business (prevalentemente relative all'area finanza, crediti e commerciale) forniscono supporto per consentire la declinazione delle eventuali azioni necessarie a garantire la continuità operativa delle funzioni critiche della Banca, delle funzioni a rilevanza sistemica e degli shared services critici. Sono inoltre informate degli orientamenti preventivamente individuati e condivisi da Risk Management, $\mathrm{CFO}$ e COO;

- la funzione Compliance valuta il rischio di non conformità connesso al processo di predisposizione del Piano di Risanamento e di attivazione e chiusura dello stato di recovery e monitora nel continuo la normativa esterna di riferimento supportando le funzioni di coordinamento nella valutazione di possibili revisioni del Piano;

- la funzione Internal Audit valuta l'adeguatezza, la fattibilità e l'efficacia del complessivo processo di recovery planning ed in particolare del processo di predisposizione del Piano di risanamento, di attivazione, di esecuzione e chiusura dello stato di recovery;

- con specifico riferimento alla calibrazione delle metriche presenti nel Recovery Plan:

- le attività di calibrazione degli scenari, misurazione degli impatti, valutazione della coerenza con le politiche di appetite/tolerance/capacity risultano prevalentemente indirizzate dal CRO che, tenuto conto delle analisi dell'evoluzione della strategia aziendale e della normativa prudenziale formula le proposte da sottoporre al Board;

- il contributo di supporto richiesto all'area CRO dalle funzioni di business per la declinazione delle azioni di recupero è prevalentemente focalizzato sull'analisi delle ricadute sull'operatività di rispettiva competenza;

- relativamente all'individuazione delle azioni di recupero il ruolo delle funzioni di business è prevalentemente focalizzato sulla discussione degli impatti degli scenari di stress sul valore residuale delle attività in situazioni di crisi e delle azioni che devono essere intraprese in caso di attivazione del piano di recupero;

- gli organi aziendali ricoprono il ruolo previsto dal dettato normativo. In particolare l'organo con funzione di supervisione strategica (tipicamente il Consiglio di Amministrazione negli enti con sistema tradizionale) valuta e approva le soluzioni identificate nell'ambito del processo, avvalendosi, ove previsto, anche del supporto di comitati aziendali (di natura endoconsiliare o gestionale). In ambito Recovery Plan è quindi chiamato a:

- approvare il processo di gestione del rischio, valutandone la compatibilità con gli indirizzi strategici e le politiche di governo dei rischi e a valutare periodicamente l'adeguatezza e la coerenza con il RAF e la compatibilità tra il rischio effettivo e gli obiettivi di rischio;

- garantire la coerenza tra il Recovery Plan e il piano strategico, il RAF, l'ICAAP, i budget e il sistema dei controlli interni (considerando anche l'evoluzione delle condizioni interne ed esterne in cui opera la Banca);

- assicurare che le scelte di allocazione del capitale e della liquidità risultino conformi agli scenari di stress individuati, quindi con la propensione al rischio dell'istituto e le conseguenti politiche di governo e di gestione dei rischi;

- garantisce, per il tramite della Segreteria Societaria, l'inoltro del Piano di risanamento approvato dallo stesso alle Autorità di Vigilanza competenti;

- l'organo con funzione di controllo (tipicamente il Collegio Sindacale negli enti con sistema tradizionale) ha, invece, la responsabilità di vigilare sulla completezza, adeguatezza, funzionalità e affidabilità del Piano di risanamento.

In Figura 3 sono sintetizzati i capisaldi individuati dal presente lavoro. 


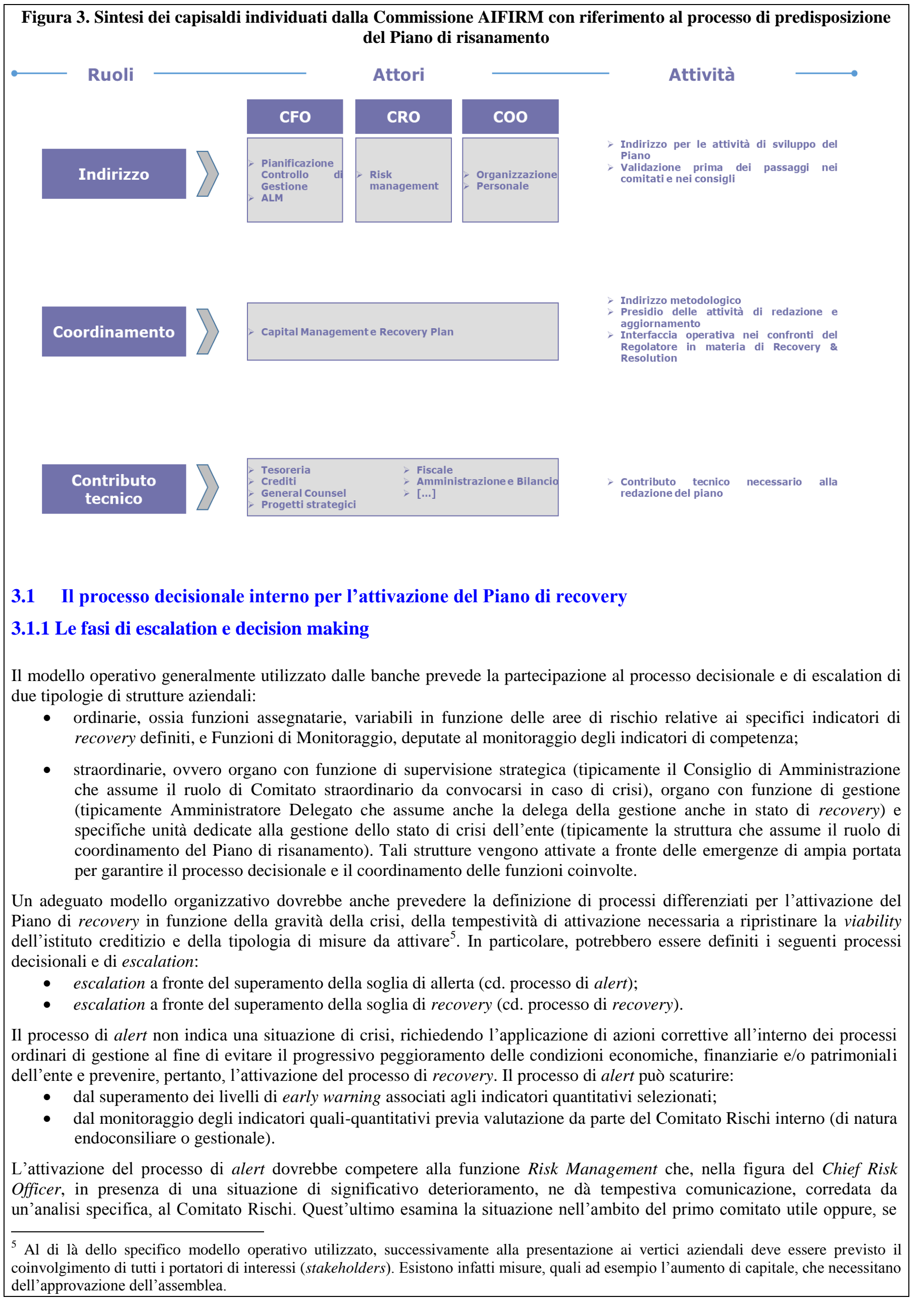


ritenuto necessario, nell'ambito di un comitato convocato ad hoc. Il Comitato Rischi, valutata la presenza di segnali di deterioramento del profilo di rischio dell'ente, potrebbe:

- attivare il processo di recovery qualora sia stata rilevata la sussistenza di una situazione di deterioramento significativo del profilo di rischio. In tal caso si deve tempestivamente provvedere a comunicare le valutazioni svolte agli opportuni organi aziendali ed eventuali ulteriori comitati interni e richiedere l'attivazione del processo di recovery da parte degli organi con funzione di supervisione strategica e di gestione;

- non attivare il processo di recovery qualora non siano state rilevate criticità. In tal caso dovrebbe essere fornita semplice informativa agli organi aziendali che, nell'ambito delle proprie competenze ordinarie, potranno intraprendere eventuali azioni correttive (cd. azioni di management intervention). In caso di Gruppi Bancari, la mancata attivazione del processo di recovery non dovrebbe dar luogo ad eventuali comunicazioni specifiche agli organi aziendali di banche e società controllate, mentre potrebbe essere prevista un'informativa attraverso i canali di comunicazione ordinari ${ }^{6}$.

Il processo di recovery, invece, può essere scomposto nelle cinque macro-fasi illustrate in Figura 4.

Figura 4. Le cinque macro-fasi distinte del processo di recovery

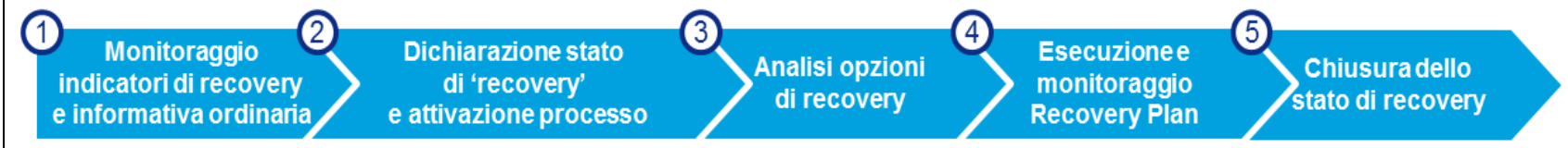

Ogni macro-fase dovrebbe essere caratterizzata da ruoli e responsabilità definiti al fine di garantire una tempestiva ed efficace reazione al manifestarsi di una situazione di deterioramento. Nello specifico:

1. monitoraggio indicatori di recovery e informativa ordinaria, ovvero attività di monitoraggio finalizzata all'individuazione tempestiva di situazioni di deterioramento significativo della situazione finanziaria;

2. dichiarazione stato di recovery, ovvero analisi delle situazioni di deterioramento rilevate attraverso le attività di monitoraggio al fine di valutarne la rilevanza e dichiarare lo stato di recovery in caso di conferma della significatività della situazione di deterioramento riscontrata;

3. analisi opzioni di recovery, ovvero selezione delle opzioni di recovery, precedentemente individuate nel Piano di risanamento, nell'effettivo contesto che si è delineato al momento della valutazione;

4. esecuzione e monitoraggio del Piano di recovery per monitorare l'attuazione delle opzioni di recovery al fine di garantire l'efficace chiusura dello stato di recovery;

5. chiusura dello stato di recovery e ripristino della situazione di normale operatività.

\subsection{Gli indicatori di recovery e il Risk Appetite Framework (RAF)}

I recovery trigger devono essere individuati coerentemente con le raccomandazioni dell'EBA e con le impostazioni del modello complessivo di Risk Appetite Framework della Banca. Tutti i recenti aggiornamenti della normativa hanno infatti enfatizzato la necessità di garantire una coerenza fra il Recovery Plan, il Risk Appetite Framework, gli indirizzi strategici e l'ICAAP. In quest'ottica, il sistema dei limiti definiti nell'ambito del RAF può rappresentare un apparato di pre-allarme, il cui superamento comporta l'identificazione degli interventi correttivi da parte del management e la loro approvazione da parte dell'organo con supervisione strategica. In questa fase le decisioni devono essere prese avendo a riferimento la strategia aziendale, che peraltro potrebbe essere adeguata per riflettere il nuovo scenario di riferimento. Percorso analogo dovrebbe avvenire con riferimento alla liquidità, nell'ambito del Contingency Liquidity Plan, che potrebbe attivarsi anche precedentemente al superamento dei limiti di RAF.

I recovery trigger sono quindi soglie minime, inferiori a quelle definite nel RAF, che identificano il momento in cui le banche dovrebbero iniziare a valutare l'attivazione del Piano di recovery in senso stretto, ovvero dello stato di crisi. Il superamento di tali soglie non comporta l'adozione automatica di tale stato, ma l'avvio della valutazione. Tra le diverse possibilità per la definizione di tali trigger, la scelta potrebbe ovviamente ricadere su quelli chiave:

- Common Equity Tier 1 Ratio (CET1\%);

- Total Tier Ratio;

- Leverage Ratio;

- Liquidity Coverage Ratio;

- Net Stable Funding Ratio.

Il Common Equity Tier 1 Ratio (CET1\%) rappresenta l'indicatore principale per la valutazione dell'adeguatezza patrimoniale nell'ambito delle regole di Basilea ed è il rapporto tra il patrimonio di primaria qualità ed il totale delle attività a rischio ponderate (Risk Weighted Asset - RWA) derivanti dai rischi tipici dell'attività bancaria e finanziaria (rischi di credito,

${ }^{6}$ D'altra parte, in caso di crisi di una società controllata devono essere attivati i canali di comunicazione straordinari con tempestiva comunicazione alla Capogruppo per concordare gli interventi correttivi necessari. Se la crisi è tale da andare a detrimento della viability del Gruppo di appartenenza, il processo di escalation dovrebbe seguire le modalità operative descritte. 
controparte, mercato e operativi). Il CET1 Ratio da adottare nell'ambito del Recovery Plan dovrebbe essere quello fully phased in, calcolato considerando già trascorso l'intero transitional period previsto da Basilea III.

Anche inserendo nella definizione del recovery gli altri due indicatori di patrimonializzazione (Total Tier Ratio e Leverage Ratio) dovrebbe essere preservata l'esigenza di semplicità, atteso che questi indicatori dovrebbero presumibilmente essere monitorati nel RAF. Soprattutto con riferimento al CET1 si sottolineano, oltre che la particolare rilevanza della misura, anche alcune proprietà tecniche dell'indicatore:

- rispetto al Leverage Ratio, il CET1 tende ad anticipare i segnali di tensione in condizione di stress. All'aumentare dei rischi, si registra infatti sia la riduzione del patrimonio sia l'incremento dei Risk Weighted Asset, mentre il Leverage Ratio presenta al denominatore un Total Asset, che tende a rimanere costante;

- rispetto al Total Tier Ratio, il CET1 è più reattivo e la sua dinamica non è influenzabile da manovre sul debito subordinato che ne possono comportare l'indebolimento del potere segnaletico;

- gli indicatori di Pillar II, infine, pur fornendo un importante punto di vista sui rischi aziendali, non risultano indicati per supportare un processo dove determinante è la rappresentazione sintetica dei rischi all'Autorità di Vigilanza.

Dal lato del rischio di liquidità, i principali indicatori quantitativi da utilizzare nel Recovery Plan possono essere gli indicatori regolamentari, ovvero il Liquidity Coverage Ratio (LCR) per la misurazione della liquidità di breve termine e l'indicatore di finanziamento stabile Net stable funding ratio (NSFR) per la liquidità strutturata.

Più in generale si sottolinea l'importanza anche di altri indicatori, non necessariamente di capitale e liquidità (peraltro presenti nelle EBA Guidelines) e si suggerisce la misurazione e il monitoraggio, ma con un diverso peso nei processi di escalation (soft trigger indicators, come ad esempio net adjusted profits, tasso di crescita dei non-performing loan, coverage ratio, tasso di variazione del PIL, CDS sovereign e banche, eventuali downgrade). La finalità è di evitare inopportune attivazioni dei piani di risanamento confinandole soltanto a casi particolarmente estremi.

In questo contesto, gli indicatori di RAF e di recovery dovrebbero rappresentare riferimenti comuni all'interno dei quali sviluppare le azioni manageriali per la gestione dell'overall risk profile delle banche. Il superamento dei limiti di RAF può rappresentare l'avvio della fase di 'Allerta' per valutare le prime azioni per ricondurre l'intermediario sul sentiero della gestione ordinaria. Analogamente, il Contingency Liquidity Plan dovrebbe assicurare l'avvio di escalation immediate ai primi segnali di crisi, quando gli indicatori di pre-allarme raggiungono determinate soglie, anche prima del superamento dei limiti di RAF. Nel caso in cui le prime azioni sul capitale e sulla liquidità non riescano ad impedire il superamento dei recovery trigger, si aprono le condizioni per la valutazione in merito all'attivazione dello stato di 'Crisi' del Recovery Plan. Il piano dovrebbe ritenersi concluso al ripristinarsi dei recovery target, ovvero nel momento in cui la Banca torna a registrare un risk profile coerente con i limiti di Risk Appetite Framework (sia per il Common Equity Tier 1 Ratio sia per il Liquidity Coverage Ratio).

Le Figure 5 e 6 forniscono una rappresentazione sintetica del processo descritto. La prima fornisce una overview semplificata delle logiche sottostanti al Recovery Plan e il collegamento con il processo RAF, la seconda presenta un framework complessivo più adatto ai grandi gruppi Bancari.

Figura 5. Indicatori di recovery e livello di trigger. Una overview semplificata delle logiche sottostanti al Recovery Plan e il collegamento con il processo $\mathbf{R A F}$

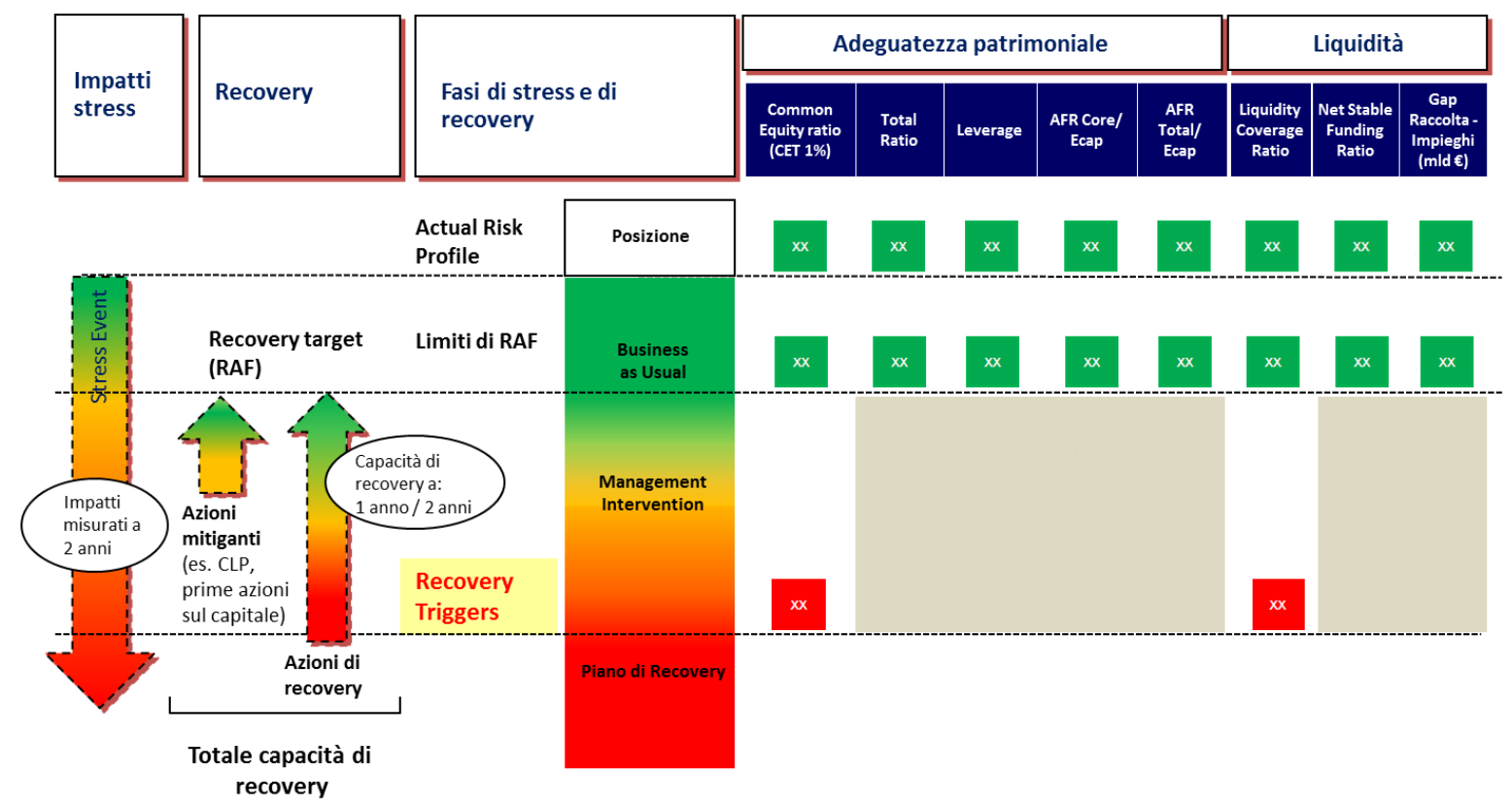




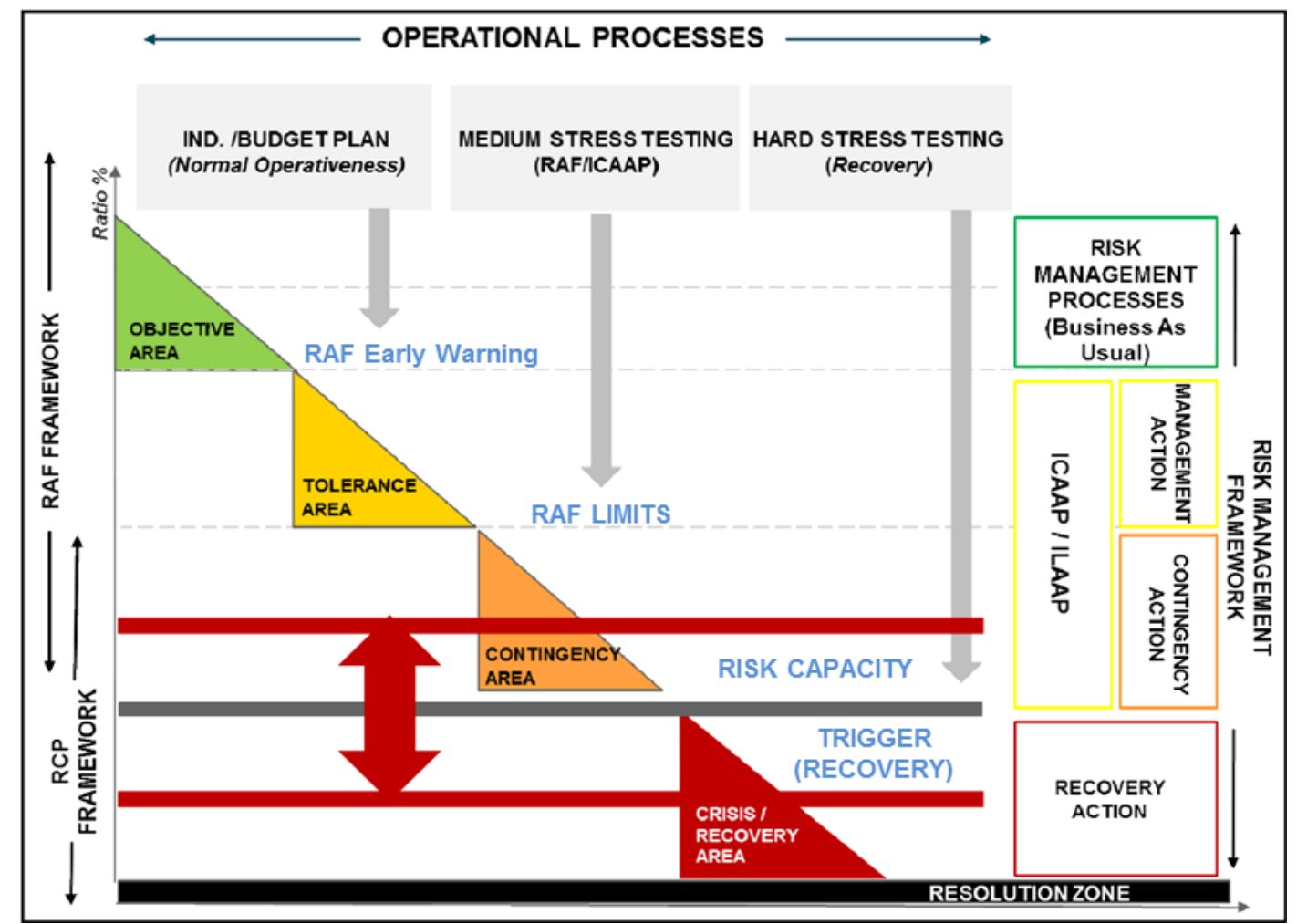

\subsection{Strategia di comunicazione e rapporti con il supervisore}

Per quanto riguarda la strategia di comunicazione in merito al Piano di recovery, è opportuno distinguere tra comunicazione con l'Autorità di Vigilanza, comunicazione esterna e comunicazione interna.

La comunicazione con l'Autorità di Vigilanza sta cambiando rapidamente. Gli approcci sono sempre più armonizzati e integrati a livello europeo e con nuovi attori sovranazionali, come il Single Supervisory Mechanism e il Single Resolution Mechanism. In tale dinamicità si evidenzia che i percorsi di comunicazione dovrebbero cambiare in base alla situazione aziendale, con procedure standardizzate in caso di business as usual ed un approccio più centralizzato in caso di crisi.

Nel caso di violazione di una delle soglie di recovery, il Chief Executive Officer dovrebbe gestire direttamente il flusso di comunicazione con il supervisore. La comunicazione iniziale dovrebbe essere trasmessa non appena tecnicamente possibile e in ogni caso entro 24 ore dalla violazione della soglia di recovery. Il messaggio di comunicazione dovrebbe includere:

- la documentazione che fornisce evidenza del superamento del trigger di recovery;

- una spiegazione delle cause dell'evento;

- la descrizione e le tempistiche delle fasi principali del processo di recupero.

Dopo la comunicazione iniziale, si suggerisce di trasmettere al supervisore i follow-up periodici sullo sviluppo della situazione e sugli effetti prodotti dalle azioni di recupero. Inoltre, non appena possibile, le comunicazioni dovrebbero essere ulteriormente integrate con il verbale del Consiglio di Amministrazione. Nel caso in cui gli indicatori di recovery mostrino un significativo miglioramento tale da revocare lo 'Stato di crisi', il Consiglio di Amministrazione, su proposta dell'amministratore delegato, dovrebbe comunicare il ritorno alla gestione ed al monitoraggio ordinari. L'annuncio al supervisore comprenderà quindi una copia della decisione del Consiglio di Amministrazione sulla revoca dello stato di crisi.

La comunicazione esterna rientra invece nell'ambito della comunicazione delle informazioni 'price sensitive' per le entità quotate (ma le logiche possono essere estese anche alle altre banche) e i soggetti che le controllano e, come tale, deve rispettare le prescrizioni della normativa riguardante le informazioni privilegiate e l'abuso di mercato. La comunicazione esterna deve avere come obiettivo ultimo una spiegazione degli accadimenti che informi gli stakeholder in merito alla solidità e alla redditività sostenibile dell'intermediario, anche al fine di consentire l'effettiva realizzazione delle azioni di recupero.

La comunicazione interna si pone infine come obiettivo quello di dare ai dipendenti tempestiva diffusione di informazioni chiare, precise ed esaurienti sulle azioni intraprese dall'azienda all'interno del piano.

\subsection{Gestione delle informazioni}

Lo studio ha ritenuto opportuno anche fornire delle indicazioni rispetto alle tematiche IT. Le informazioni necessarie per la costruzione del Piano di recovery dovrebbero essere prevalentemente acquisite dai sistemi applicativi di governo e di sintesi della Banca. Le informazioni necessarie alla stesura del piano e all'esecuzione delle azioni di recovery dovrebbero essere costituite principalmente da estrazioni mirate richieste dai vari gruppi di lavoro provenienti da: 
- $\quad$ sistemi informativi di governo e di sintesi;

- accessi ad applicativi specifici a disposizione delle singole funzioni della Banca che hanno partecipato alla stesura del piano;

- documenti ufficiali già prodotti e disponibili (es. le relazioni semestrali).

Nell'ambito dei sistemi informativi direttamente gestiti, le policy di data management dovrebbero essere suddivise nei seguenti filoni tematici:

- Data Governance e Ownership: tutte le informazioni (dati) presenti nel sistema hanno un owner tecnico e un corrispettivo owner di business identificati e responsabili di ogni evoluzione sulle informazioni gestite.

- Data Structure e Metadata Management: in quest'ambito rientrano policy, strumenti e metodi relativi alla modellazione e alla gestione della tassonomia delle informazioni. Le strutture IT dovrebbero definire standard trasversali per tutto il sistema informativo.

- Data Architecture \& Security: rientrano in questo ambito le policy relative alle modalità di accesso tecnico alle informazioni, le policy di migrazione dei dati, di gestione dello storage e le policy di backup e archiviazione.

- Master Data Management: i master data a cominciare prima di tutto dalle informazioni relative alla clientela e i prodotti e rapporti che vengono governati. Si pone particolare attenzione al fatto che le informazioni non siano ridondate tra sistemi differenti e ove necessario per ragioni tecniche e di performance si dovrebbe mantenere traccia dei sistemi sorgenti (master).

- Data Quality: dovrebbero esistere specifiche policy, processi e strumenti di data quality. I processi di data quality dovrebbero essere coordinati e governati da una cabina di regia che governa le segnalazioni di anomalia per i diversi utilizzatori delle informazioni presenti nel sistema informativo.

L'unità a presidio del Recovery Plan dovrebbe avere cura di avviare un piano di progetto per la realizzazione dell'infrastruttura tecnologica 'Data Room' il cui obiettivo dovrebbe essere quello di guidare la produzione, la raccolta e la fruibilità dei dati e delle informazioni necessarie per l'aggiornamento del Piano di recovery e per la redazione del Resolution Plan. In tal senso la realizzazione della Data Room dovrebbe essere effettuata in sinergia con i progetti IT esistenti, allineandosi con le scelte tecnologiche e con il piano delle attività realizzative previste.

Al contempo, tale infrastruttura dedicata dovrà garantire, nella produzione e nella fornitura delle informazioni, standard qualitativi dei dati in linea con le prassi e gli schemi informativi della Banca, del mercato e secondo quanto stabilito dall'Autorità di Vigilanza.

In Figura 7 viene dettagliata una esemplificazione della struttura informativa necessaria alla stesura del piano e all'esecuzione delle azioni di recovery. Dovrebbe trattarsi, in generale, di estrazioni di dati provenienti dai sistemi informativi già esistenti, di documenti ufficiali prodotti nel continuo dalla Banca (es: le relazioni semestrali) e di accessi ad applicativi a disposizione delle singole funzioni dell'intermediario che partecipano alla stesura del piano.

Figura 7. Struttura informativa necessaria alla stesura del piano e all'esecuzione delle azioni di recovery

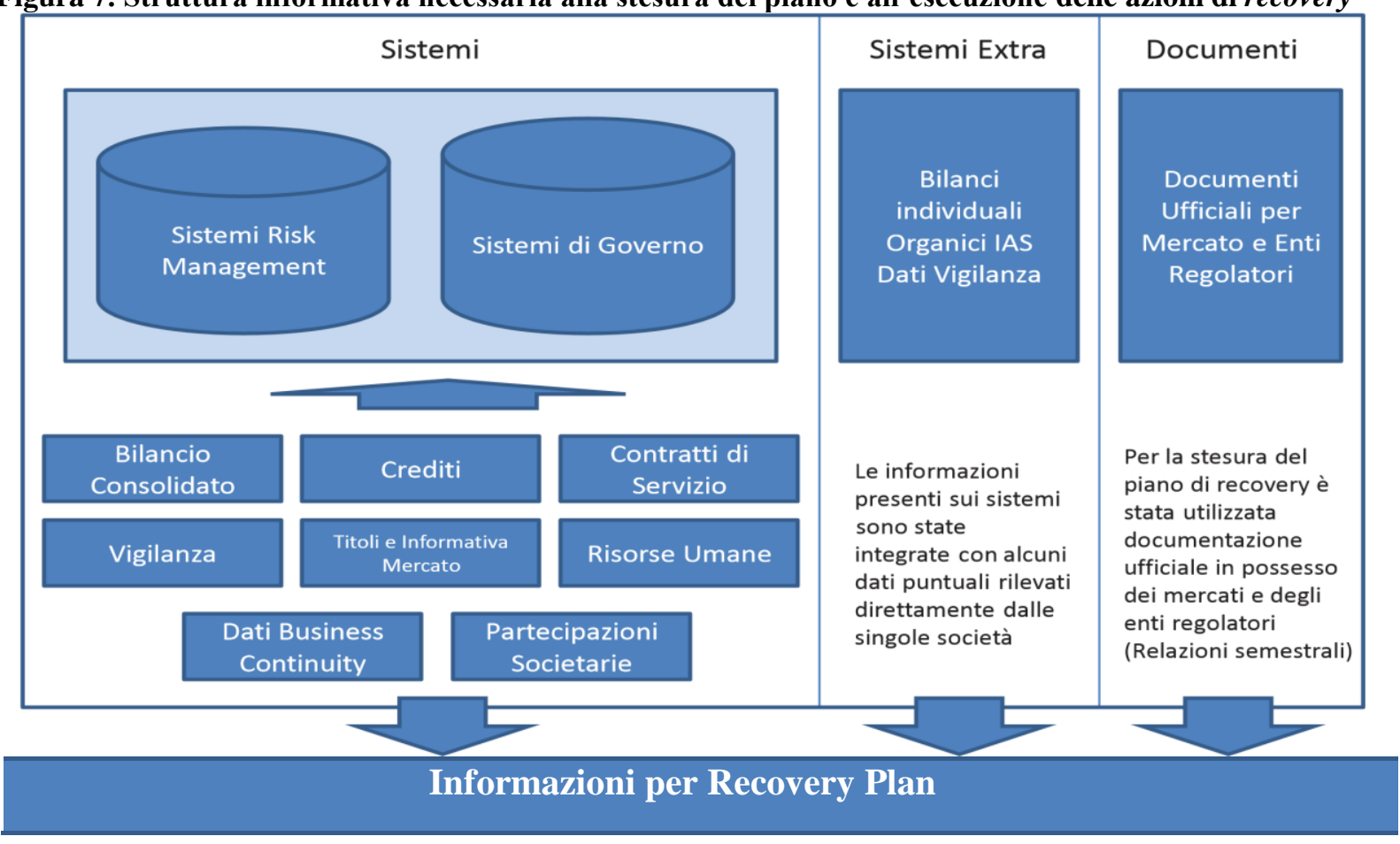




\section{Scenari di stress}

\subsection{Reverse Stress Testing}

In considerazione dell'esperienza europea degli ultimi anni e delle conseguenti aspettative da parte del Regulator sulle attività di stress testing da parte delle Banche, l'EBA nel 2016 ha pubblicato il Consultation Paper "EBA/CP/2016/28 - Draft Guidelines on stress testing and supervisory stress testing". Tale documento ha lo scopo di promuovere la convergenza in termini di approccio da parte sia delle Banche che delle competenti Autorità nazionali.

In tale contesto si inquadrano anche le raccomandazioni dell'EBA nell'ambito Recovery Plan. L'adozione di scenari di stress specifici per singola Banca in grado di comprometterne la solvibilità dovrebbe basarsi su un approccio di derivazione inversa ovvero identificando la situazione in cui la stessa può essere considerata 'failing or likely to fail' a causa di un deterioramento della situazione patrimoniale e/o del profilo di liquidità (cd. 'Reverse Stress Test').

Il Reverse Stress Testing è volto ad incrementare la consapevolezza della Banca riguardo alle proprie aree di vulnerabilità mediante l'attività di esplicita identificazione e valutazione degli scenari di severità tale da pregiudicarne la stessa resilienza. Tale approccio permette anche alla Banca di identificare preventivamente le tipologie di azioni manageriali e le relative tempistiche di attuazione, al fine di mitigare e gestire possibili criticità legate al proprio modello di business.

L'identificazione degli scenari di Reverse Stress Test viene eseguita in considerazione dell'operatività della Banca, della propria capacità di generare profitti, del proprio modello di funding, della propria struttura organizzativa nonché del potenziale impatto di eventi di natura idiosincratica e sistemica ${ }^{7}$. Vengono pertanto identificati diversi scenari, assicurandone la coerenza con lo scenario di base (adottato ai fini delle attività di planning della Banca) ed applicando ipotesi avverse riguardo ai principali fattori macroeconomici, di mercato ed idiosincratici.

\subsection{Processo di selezione degli scenari}

Come sopra specificato, la selezione degli scenari di rischio della Banca deve considerare diverse aree di indagine al fine di garantire l'identificazione e la completezza della misurazione di tutti i rischi materiali, sulla base ad esempio dei seguenti elementi:

- $\quad$ il quadro macroeconomico generale, sia a livello nazionale sia internazionale, ed i relativi scenari di rischio, che possono determinare significative vulnerabilità del valore dell'attivo e del profilo di rischio dell'intermediario;

- $\quad$ il confronto periodico con gli analisti delle società di rating, survey/reports in tema di rischi prospettici predisposti da diversi organi internazionali (BIS, CEBS/EBA, BCE e Financial Stability Board per citare quelli più significativi);

- le discussioni emergenti dai diversi comitati dell'intermediario, dove la situazione attuale e prospettica dei rischi nei diversi ambiti viene analizzata dai vari esperti aziendali, mettendo a fattor comune i diversi punti di vista;

- gli esercizi di stress test, sia quelli condotti internamente nell'ambito della definizione dei processi Risk Appetite Framework/ICAAP o ogni volta se ne presenti la necessità a fronte di una potenziale minaccia, sia quelli condotti su richiesta dell'autorità di vigilanza;

- lo stato patrimoniale ed il conto economico attuale e prospettico in quanto costituenti in primis ambito di indagine per l'identificazione dei rischi (la valutazione si estende su tutte le poste patrimoniali ed economiche delle diverse aree di business e delle principali entità giuridiche);

- le indicazioni normative previste a livello nazionale, nonché le best practices internazionali;

- $\quad$ i processi di collocamento di nuovi prodotti in quanto ogni nuovo prodotto trattato, per esigenze della proprietà o della clientela, deve essere oggetto di un articolato sistema di assessment nell'ambito del quale vengono ad essere individuati e gestiti eventuali nuovi rischi rilevanti;

- $\quad$ i processi e le procedure organizzative, che sono periodicamente soggetti a valutazione da presidi decentrati, al fine di mitigare l'insorgere dei rischi operativi.

La selezione degli scenari deve quindi mirare ad identificare quelli che maggiormente incidono sulle principali vulnerabilità della Banca. In particolare, devono essere valutati, tra gli altri, eventi severi ma plausibili che possono impattare il modello di business, la struttura del funding plan e le interconnessioni con il sistema finanziario.

Come richiesto dal regolatore, per colpire queste vulnerabilità devono essere ipotizzati diversi scenari di stress che contemplano eventi sistematici ed idiosincratici caratterizzati sia da eventi repentini ed immediati (fast movement) sia da altri a progressiva e lenta materializzazione (slow movement). La scelta degli scenari è legata all'obiettivo di raggiungere il punto di 'likely to fail', finalità perseguibile anche con combinazioni di eventi particolarmente estremi al limite della plausibilità.

A tale scopo sono identificate tre tipologie di eventi:

- evento idiosincratico, rappresentato da un evento che può comportare conseguenze negative per il gruppo o singole società controllate;

- evento sistemico, rappresentato da un evento che può comportare conseguenze negative per il sistema finanziario o l'economia reale nel suo complesso;

${ }^{7}$ Lo strumento di generazione degli scenari macroeconomici dovrebbe garantire la produzione di un elevato numero di percorsi evolutivi che descrivano le principali variabili macroeconomiche nel rispetto del principio di plausibilità. A tale scopo, viene generalmente adottata una metodologia che prevede l'utilizzo di un modello macroeconomico che analizza l'economia globale, con particolare riferimento alle relazioni finanziarie e le interazioni macroeconomiche tra diverse economie nazionali in una prospettiva globale, focalizzandosi anche sui legami tra finanza pubblica e tassi di interesse. Costituisce un esempio di tale modello il Global Vector AutoRegressive Model introdotto da Pesaran et al. (2004). 
- evento combinato, rappresentato da una combinazione di eventi di tipo sistemico e idiosincratico che si verificano simultaneamente ed in maniera interrelate.

A titolo esemplificativo, nella definizione degli scenari sistemici può essere tenuta in considerazione la combinazione di differenti eventi, quali:

- $\quad$ recessione economica sui principali mercati di riferimento;

- $\quad$ fallimento di importanti controparti con potenziali effetti sulla stabilità del sistema finanziario;

- $\quad$ crisi di liquidità del mercato interbancario;

- $\quad$ incremento del rischio paese su uno mercato in cui la Banca è esposta;

- $\quad$ riduzione generalizzata del valore degli attivi di bilancio;

Con riferimento allo scenario idiosincratico, possono essere implementati i seguenti eventi di crisi:

- $\quad$ fallimento di principali controparti cui la Banca è esposta;

- evento che determina un danno reputazionale, ad esempio attacco cyber che porta alla perdita di dati sensibili della clientela;

- $\quad$ significativo deflusso di liquidità;

- $\quad$ riduzione del valore degli attivi specifici per la Banca;

- importanti perdite sui crediti dovuto al deterioramento del portafoglio della Banca o su un segmento cui la Banca è particolarmente esposta;

- $\quad$ importante perdita operativa.

In sintesi, gli scenari di stress devono permettere di testare efficacemente la capacità e l'adeguatezza degli indicatori selezionati di rappresentare tempestivamente il deterioramento della Banca e l'efficacia e l'adeguatezza delle recovery option adottate.

\section{Azioni di Recovery}

Un'altra componente cruciale del Piano di recovery è rappresentata dall'individuazione delle azioni di recovery, vale a dire le azioni da attuare in virtù della loro potenziale capacità di ripristinare la solidità della Banca in situazioni di stress (ripristinando ad es. i livelli indicati nel Risk Appetite Framework). Le azioni devono essere presentate in dettaglio, con esposizione dei razionali di scelta, dell'eventuale esperienza pregressa già maturata, della fattibilità, degli impatti finanziari, operativi interni ed esterni, e del processo di execution.

Le azioni di recovery possono essere classificate in due macro categorie:

1. azioni tattiche mitiganti volte a contenere, nell'immediato, l'effetto di eventi specifici previsti in alcuni scenari;

2. azioni di recovery, descritte analiticamente nel corso della trattazione.

Le azioni tattiche mitiganti sono da mettere in relazione ai singoli scenari ipotizzati e rappresentano le azioni che verrebbero immediatamente poste in essere come prima risposta al verificarsi degli scenari. Sono generalmente individuate in tre sottocategorie:

A. annullamento dei dividendi;

B. mantenimento inerziale fino a scadenza del portafoglio titoli governativi FVTOCI e FVTPL con vita residua compresa tra 6 e 12 mesi. Il mantenimento fino a scadenza dei titoli governativi classificati FVTOCI e FVTPL, a meno di ipotesi di default sul rischio sovrano, consente di evitare gli haircut relativi al primo anno dell'orizzonte di analisi;

C. perdita del ruolo di account bank: nei programmi OBG (Obbligazioni Bancarie Garantite) sono previste soglie di rating al di sotto delle quali la Banca cessa di essere controparte idonea per il mantenimento dei conti correnti dei veicoli. In tali circostanze è attivabile un'azione mitigante consistente nel parziale rimborso dei finanziamenti subordinati concessi dalla Banca al veicolo (finalizzati a finanziare il pagamento degli asset da parte del veicolo).

Per quanto riguarda le azioni di recovery vere e proprie, le banche hanno a disposizione un ampio ventaglio di opzioni che impattano su una molteplicità di voci dell'attivo e del passivo. Da un punto di vista logico le azioni individuate sono riconducibili a cinque macro categorie:

1. riduzione attivi di bilancio;

2. cessione partecipazioni di minoranza;

3. cessione partecipazioni di maggioranza;

4. generazione straordinaria di liquidità;

5. azioni sul patrimonio.

Per ciascuna azione e sotto-azione, devono essere stati valutati gli impatti finanziari e la congruità nei diversi scenari di stress, in termini di azionabilità, impatti interni ed esterni e fattibilità. Il risultato delle analisi deve offrire una view sull'effettiva capacità di recupero delle azioni. In particolare:

- fattibilità: deve essere definita una descrizione del grado di fattibilità dell'operazione, con particolare riferimento alla capacità di rispetto delle tempistiche, di gestione del processo, alle ipotesi sul contesto di mercato in cui viene eseguita la misura e all'attrattività della cessione per tipologia di scenario. Deve inoltre essere realizzata una sintesi 
dei principali ostacoli e rischi che potrebbero ostacolare/rendere meno efficaci le azioni di recovery nei differenti scenari. Infine deve essere verificata la complessiva sussistenza di condizioni della loro fattibilità anche in condizioni di applicazione simultanea delle azioni;

- impatti finanziari: devono essere calcolati gli impatti sui ratio patrimoniali (CET1 Ratio e/o altri coefficienti) e sulla liquidità (Liquidity Coverage Ratio e/o altri indicatori) in funzione e in coerenza degli scenari;

- $\quad$ impatti operativi interni ed esterni: devono essere valutati gli impatti operativi interni (ad es. su attività di business core, sull'organizzazione) ed esterni, con particolare attenzione alle interazioni tra azioni e contesto in cui vengono attuate;

- misure preparatorie: devono essere analizzate le eventuali misure preparatorie volte a superare possibili impedimenti o rallentamenti all'execution delle azioni di recovery.

Ove significativo, dovrebbero infine essere effettuate analisi di sostenibilità dal lato sia reddituale sia del profilo di rischio post esecuzione delle azioni di recovery.

Considerando tutte le azioni di recovery esercitabili (con e senza alcun aumento di capitale), le analisi dovrebbero essere integrate con:

A. Impatti sulla Profittabilità. L'analisi deve focalizzarsi sul risultato dell'esercizio di tutte le azioni disponibili. In particolare, il risultato deve informare sulla capacità o meno di generare utili nel lungo termine.

B. Impatti sul Risk Profile. La valutazione deve riguardare anche l'adeguatezza patrimoniale di Pillar I e Pillar II: il CET1 ratio finale deve essere confrontato con i livelli di RAF, ispirati come intuibile dagli obiettivi di rating della Banca.

\section{IFRS 9 e implicazioni per il Piano di Recovery}

Un altro spunto di riflessione deriva dall'adozione del nuovo principio contabile internazionale IFRS 9, che rappresenta una delle maggiori sfide degli ultimi anni per le banche italiane. Il nuovo standard contabile, emanato nel luglio 2014 dallo IASB, è entrato in vigore il $1^{\circ}$ gennaio 2018 con significative differenze rispetto a IAS 39. Filo conduttore del nuovo principio è l'evoluzione delle metodologie di valutazione delle rettifiche di valore per il rischio creditizio. Il nuovo modello di impairment ha infatti trasformato le ormai consolidate pratiche bancarie, incentrate su un'ottica incurred loss, in un approccio basato sull'expected loss che tiene in considerazione anche gli effetti degli scenari macroeconomici attesi (cd. misure forward looking).

In estrema sintesi, le principali novità introdotte dal principio ${ }^{8}$ possono essere descritte come di seguito:

- introduzione di tre specifici business model, cui ricondurre i modelli contabili per la gestione delle attività finanziarie:

a) hold to collect (HTC), per cui le attività sono misurate in base al costo ammortizzato (CA);

b) hold to collect and sell (HTCS), per cui le attività finanziarie sono valutate al fair value con impatto sulla redditività complessiva (FVTOCI);

c) modello residuale ('Other' che include il trading), per cui le attività finanziarie sono valutate al fair value con impatto a conto economico (FVTPL);

- introduzione di un test, a livello di singolo strumento finanziario, volto a verificare che le caratteristiche contrattuali siano riconducibili a quelle di un 'basic lending arrangement' (cd. solely payment of principal and interest - SPPI). Le attività con caratteristiche contrattuali diverse da quelle SPPI devono essere obbligatoriamente valutate al fair value con variazioni imputate a conto economico (FVTPL);

- determinazione delle rettifiche di valore su crediti in bonis e deteriorati, sulla base del modello di Expected Credit Losses (ECL), che includono informazioni prospettiche (forward looking) anche di tipo macroeconomico;

- $\quad$ introduzione di differenti stadi (staging) per la classificazione dei crediti, cui corrispondono rettifiche di valore basate su ECL a 12 mesi (Stage 1), ovvero 'lifetime' per tutta la durata residua dello strumento (Stage 2 e 3), sulla base del Significativo Incremento del Rischio di Credito (SICR) determinato tramite il confronto tra le probabilità di default alla data di prima iscrizione e alla data di bilancio.

Risulta evidente quanto siano materialmente profonde le azioni poste in essere dai Gruppi Bancari per implementare la nuova normativa contabile. Il calcolo degli impairment ha richiesto lo sviluppo di un framework metodologico completamente nuovo per la stima dei parametri di rischio che ha coinvolto il Risk Management e diverse funzioni aziendali con risultati che sono sintetizzati dai notevoli impatti derivanti dalla First Time Adoption (FTA). Molto importanti saranno presumibilmente anche le implicazioni per il pricing del nuovo business.

Per quanto riguarda i Piani di recovery, l'introduzione del principio non ne sconvolge la struttura di fondo, anche se potrebbe avere conseguenze nel modo di creare gli scenari, sugli indicatori prospettici, sulle azioni di recovery e ovviamente sui meccanismi di governance. Infatti, il livello di sofisticazione e gli investimenti richiesti dell'impianto metodologico proposto per il rischio di credito risultano ormai ad un elevato stato di evoluzione da non potersi limitare ad esercizi meramente regolamentari, presupponendo un nuovo modello operativo dove si rende necessaria una maggiore collaborazione tra le aree CFO e CRO. Visto che le stime degli accantonamenti, in precedenza solo di competenza del CFO, richiederanno sempre più

${ }^{8}$ Per ulteriori approfondimenti si rimanda al Position Paper AIFIRM n. 8 "Il principio contabile IFRS 9 in Banca: la prospettiva del Risk Manager" - dicembre 2016. 
un continuo contributo da parte del Risk Management, è prevedibile che le banche (non solo quelle di maggiori dimensioni) mettano in cantiere lo sviluppo di sistemi informativi capaci di mettere simultaneamente in comunicazione le varie aree aziendali.

Con riferimento alle attività impattate nell'area CFO, possiamo includere le seguenti:

- $\quad$ pianificazione strategica;

- $\quad$ budget e sistema premiante;

- $\quad$ planning portafoglio creditizio (rischio/rendimento - strategie creditizie).

Dal punto di vista dell'area CRO, i processi impattati sono quelli più vicini alla governance aziendale, ovvero:

- RAF e in particolare il 'cascading' sul portafoglio crediti;

- $\quad$ stress test ICAAP e regolamentare;

- Recovery Plan, scenari di Reverse Stress Test (BRRD) e azioni di recovery.

Tali necessità di integrazione risultano ulteriormente enfatizzate dalle indicazioni dell'EBA sull'Institution's Stress Testing Programme (estendibile a tutte le realtà con il principio di proporzionalità). La European Banking Authority ha infatti pubblicato il 18 dicembre 2015 il Consultation Paper Draft Guidelines on stress testing and supervisory stress testing (EBA/CP/2015/28), seguito il 31 ottobre 2017 dal Consultation Paper on Guidelines on Institution's Stress Testing (EBA/CP/2017/17), allo scopo di favorire maggiore omogeneità degli approcci seguiti dalle banche europee in tutte le attività di stress testing e quindi anche in ambito Recovery Plan. Tali documenti, una volta pubblicati in versione definitiva, forniranno indicazioni dettagliate che le banche saranno tenute a rispettare nel predisporre l'operatività ordinaria e straordinaria, non soltanto quindi sotto un profilo prettamente metodologico. Il 31 ottobre 2017 l'EBA ha pubblicato anche un Consultation Paper on Guidelines for Common Procedures and Methodologies for SREP (EBA/CP/2017/18), anch'esso rilevante per indirizzare il percorso evolutivo del business degli intermediari finanziari.

In sintesi, il principio IFRS 9 costituisce un'opportunità per una maggiore integrazione CFO e CRO (e in generale dei processi di governo) dato che:

- $\quad$ il principio IFRS9, sia in ottica statica che di pianificazione, implica condividere modelli, scenari, sensitivities, ma soprattutto la cultura e le tecniche del Risk Management;

- risulta necessario avere strutture dedicate e flessibili con un forte orientamento al risultato, ma con un solido 'backbone' di integrated data quality;

- tali esigenze sono essenziali per tutte le banche (non solo per quelle di maggiori dimensioni), soprattutto nell'ottica di dialogo fra i vari processi ICAAP, RAF e Recovery Plan a fini SREP.

\section{Conclusioni: spunti di riflessioni e nuove opportunità}

Nel presente articolo sono stati indicati i principali punti di criticità in materia di recovery, tenendo conto delle prassi di mercato oltre che delle richieste regolamentari. Dai risultati della sopra citata survey AIFIRM su 13 Istituti Bancari italiani di diverse dimensioni (6 vigilati direttamente dalla BCE e 7 da Banca d'Italia), è emerso un quadro variegato in termini di grado di maturità e di differenze nell'approccio utilizzato. In particolare in taluni ambiti sono state riscontrate delle significative convergenze, per esempio riguardo alle tipologie e al numero di opzioni considerate, agli indicatori inclusi nei rispettivi piani di risanamento.

\section{Figura 8. Indicatori di Capitale.}

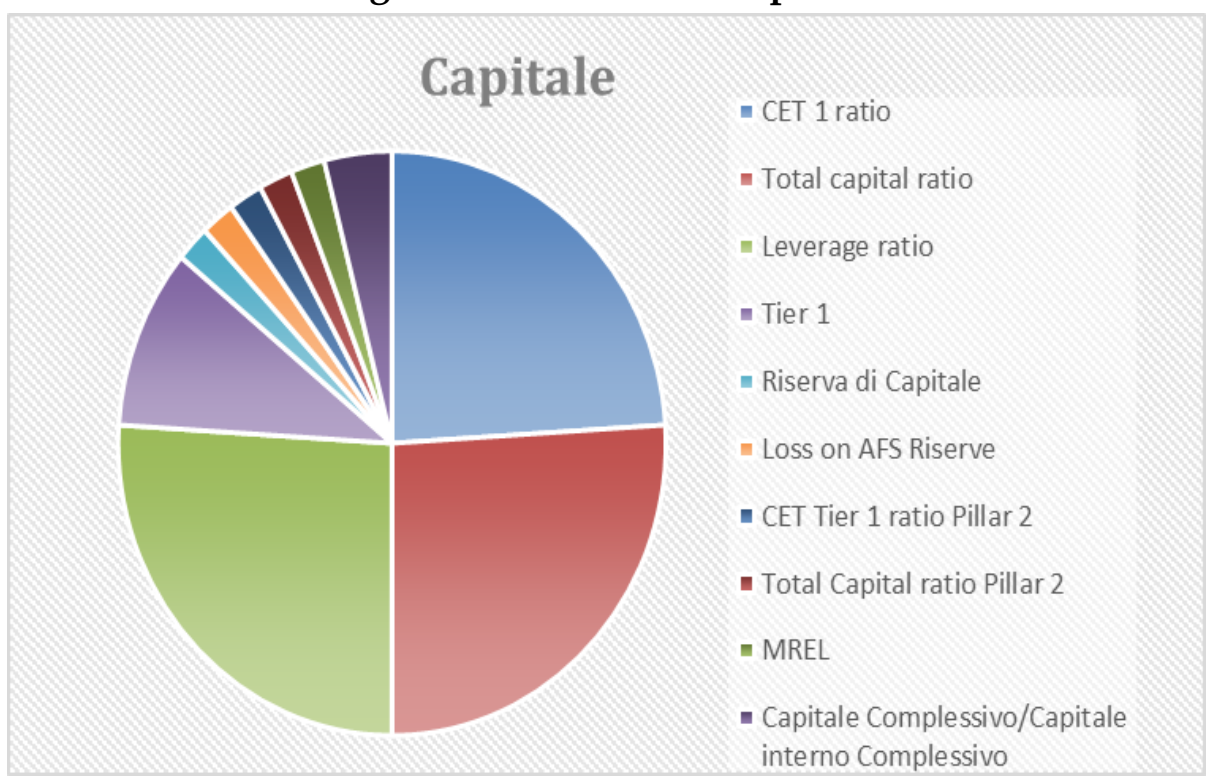


Figura 9. Capital Raising Options.

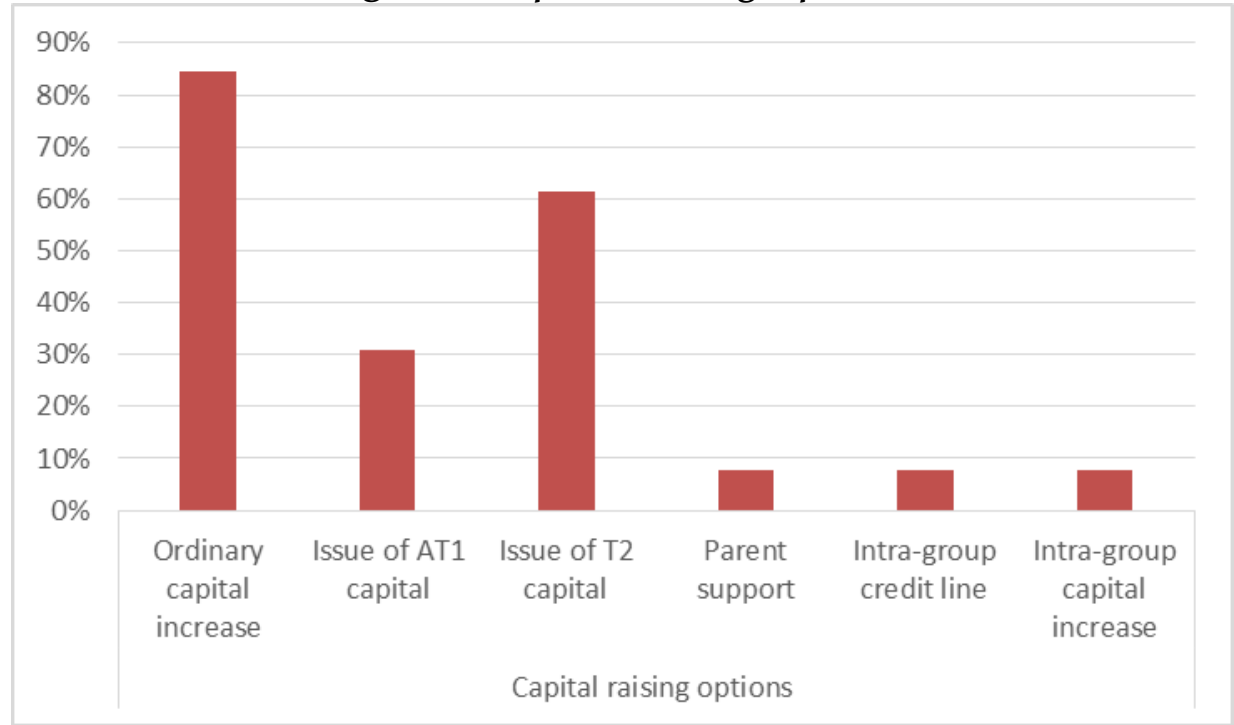

Figura 10. Disposal.

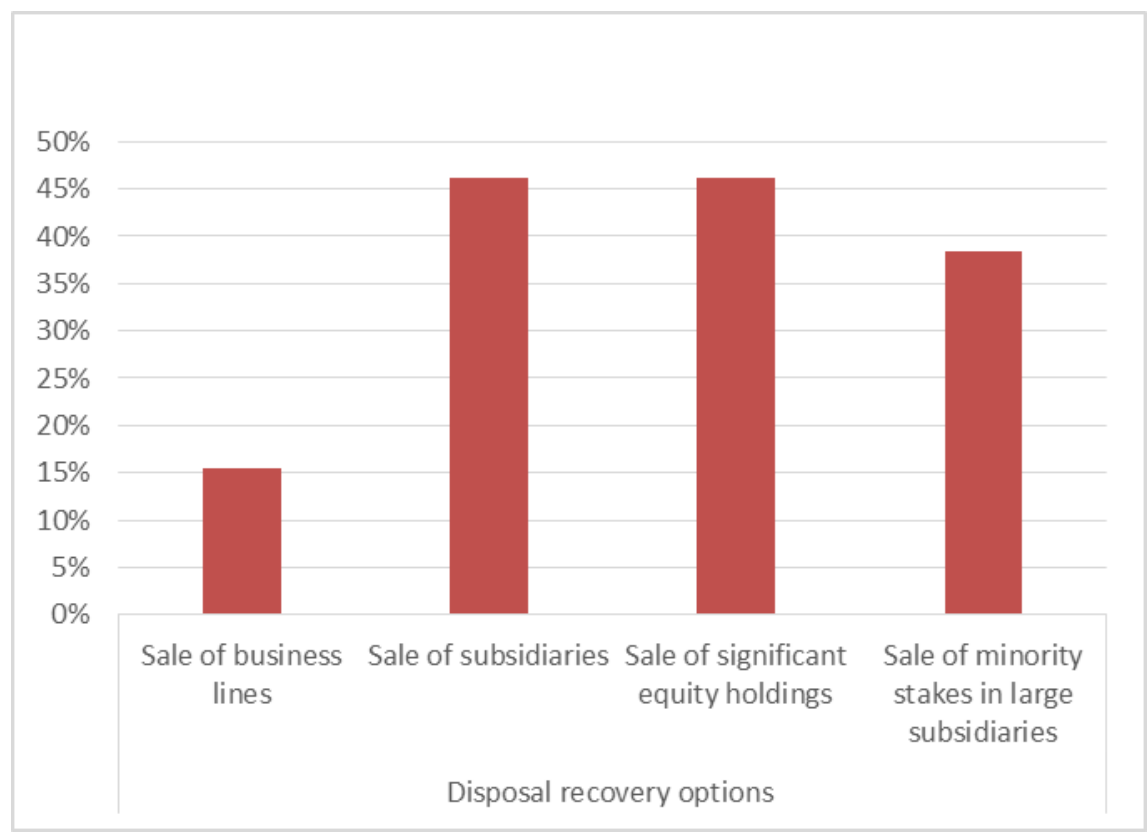

In altri ambiti, invece, sono state evidenziate differenze d'impostazione, ad esempio in relazione ai ruoli e alle responsabilità delle varie funzioni rispetto ai processi di recovery o in relazione al coordinamento con altri processi di governance, come $\mathrm{i}$ processi di escalation o le modalità di calibrazione degli indicatori di recovery, etc.

Con riferimento agli scenari di stress, le banche hanno incluso, coerentemente con le vulnerabilità connesse al proprio business model e quindi alla rilevanza degli scenari di stress rispetto alle caratteristiche dell'istituto, ulteriori scenari oltre a quelli suggeriti dall’EBA. 
Figura 11. Scenari idiosincratici maggiormente utilizzati dalle Banche oggetto d'analisi.

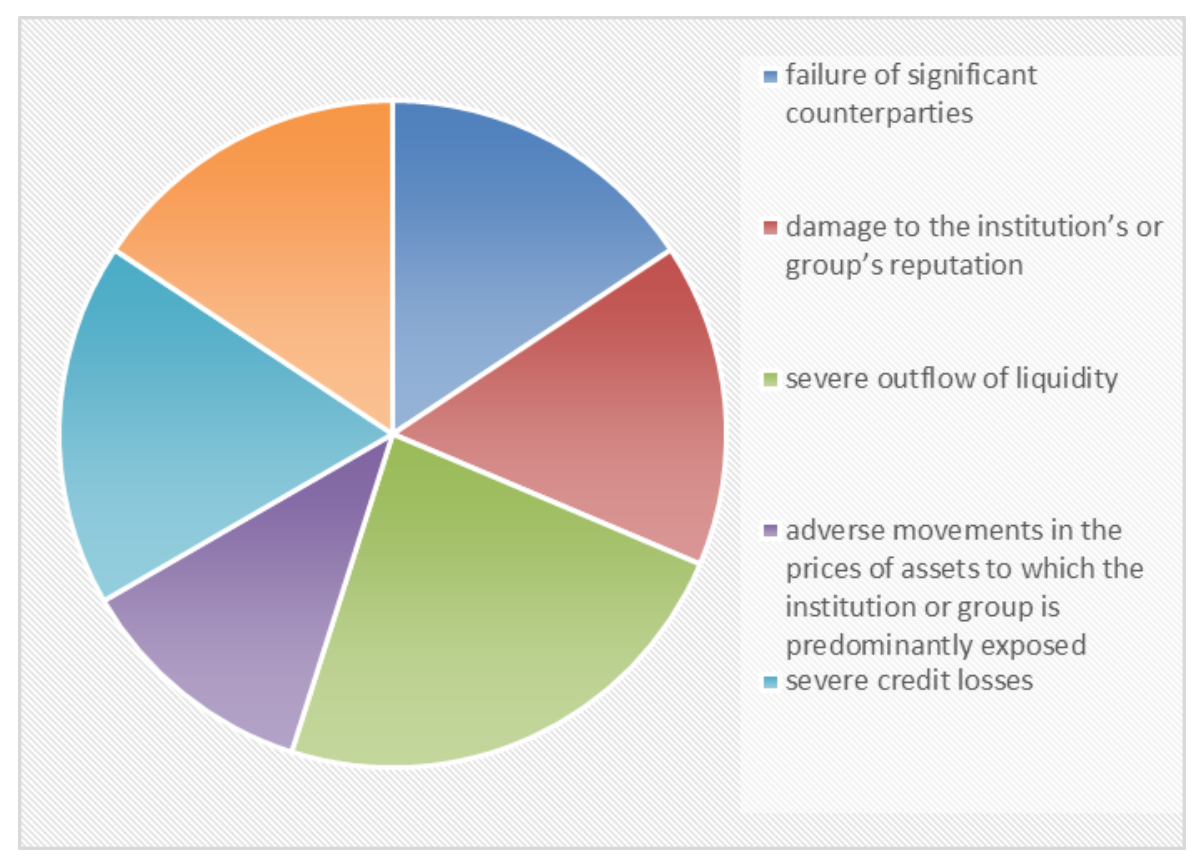

In generale, in considerazione del fatto che è lecito attendersi una costante evoluzione in materia di recovery che tenga conto delle modifiche interne alla Banca e del contesto competitivo esterno, è possibile identificare alcuni ambiti di potenziale miglioramento che potrebbero essere affrontati dalle banche italiane nei prossimi anni, in particolare:

- integrazione del Piano di risanamento con altri processi aziendali;

- perimetro degli indicatori;

- calibrazione degli indicatori;

- rilevanza degli scenari di stress rispetto alle caratteristiche dell'istituto;

- coerenza tra scenari di stress rispetto agli esercizi di stress già utilizzati in ambito Risk Management;

- perimetro delle opzioni di risanamento;

- analisi di fattibilità delle opzioni di risanamento;

- Management Information System (MIS);

- politiche e procedure.

- passaggio da un approccio risk tolerance (anche evoluto con i limiti stabiliti nel RAF/Recovery) ad uno wide comprehensive in cui gli scenari di stress vengono utilizzati per l'ottimizzazione rischio/rendimento;

- prioritizzazione delle azioni di recovery in modo da valutare attentamente le ricadute sul sistema dell'economia reale;

- rafforzamento della disclosure interna e del coordinamento nella gestione del piano;

- analisi delle opzioni di risanamento rispetto agli scenari di stress identificati.

Come conseguenza le priorità di sviluppo dei prossimi anni potrebbero essere:

- rafforzamento dei processi di capital allocation in coerenza con l'ottica RAF;

- relazione con i processi di budgeting;

- declinazione delle metriche in modo non top-down;

- sviluppo maggiormente integrato con Piano di resolution;

- maggiore disclosure sui criteri di assessment (peer benchmarking e comparability analysis disclosed);

- approfondimento delle modalità di esecuzione delle opzioni di recovery al fine di individuarne possibili impedimenti ed ostacoli operativi e legali - sia a livello di gruppo sia a livello di singoli enti cui il piano si rivolge - nonché le relative azioni per il loro superamento;

- maggiore applicazione del principio di proporzionalità, in particolare nella definizione degli stress test;

- miglior definizione del processo di governance e delle soglie in modo tale che il tema del risanamento sia effettivamente a tutela della stabilità del sistema creditizio e non diventi, invece, una possibile fonte esso stesso di tensioni e incertezze.

Alla luce delle considerazioni e degli spunti di riflessione esposti, si auspica che gli elementi oggetto di analisi e le conclusioni sopra sintetizzate costituiscano, da un lato un utile contributo operativo per le banche impegnate nell'identificare ed apportare gli opportuni correttivi di breve termine ai rispettivi Piani di risanamento e, dall'altro, un quadro di riferimento per indirizzare l'evoluzione del Recovery Plan alla luce delle esperienze osservate nelle best practices bancarie e dell'interlocuzione con le Autorità di Vigilanza, al fine di consolidarne il ruolo e la centralità all'interno della governance aziendale.

Fabio Verachi 


\section{Bibliografia}

AIFIRM (2018), Recovery Plan: punti di forza e di debolezza

I coordinatori della Commissione Aifirm:

Mario Anolli, Maurizio Vallino, Fabio Verachi

I contributori ai lavori:

Antonio Della Valle, Marco Paonessa, Enrica Pennetta, Blerta Pupe, Salvatore Spagnolo

I referenti accademici per i tavoli di lavoro che si erano costituiti ai fini dello studio

Marina Brogi e Anna Grazia Quaranta

e tutti i partecipanti alla Commissione

Pubblicazioni citate nel lavoro:

- Consultation paper on Guidelines on institution's stress testing, pubblicato il 31 ottobre 2017;

- Guidelines on the range of scenarios to be used in recovery plans, publicate il 18 luglio 2014;

- Guidelines on the minimum list of qualitative and quantitative recovery plan indicators, publicate il 6 maggio 2015;

- Guidelines on the specification of measures to reduce or remove impediments to resolvability and the circumstances in which each measure may be applied under Directive 2014/59/EU, pubblicato a novembre 2014;

- Guidelines on common procedures and methodologies for the supervisory review and evaluation process (SREP), pubblicate il 19 dicembre 2014;

- Guidelines on ICAAP and ILAAP information collected for SREP purposes, pubblicate il 19 dicembre 2014;

- RTS on the content of recovery plans under Article 5(10) of Directive 2014/59/EU establishing a framework for the recovery and resolution of credit institutions and investment firms, pubblicato il 4 febbraio 2016;

- $\quad$ RTS on the assessment of recovery plans under Article 6(8) of Directive 2014/59/EU, pubblicato il 23 marzo 2016;

- Comparative report on governance arrangements and recovery indicators, pubblicato il 5 luglio 2016;

- Comparative report on scenario in recovery plans laying down the approaches taken by a sample of European banking groups on scenarios in recovery plans, pubblicato il 8 dicembre 2015;

- Comparative report on governance arrangements and recovery indicators pubblicato il 5 luglio 2016;

- Comparative report on the approach to determining critical functions and core business lines in recovery plans, pubblicato il 6 marzo 2015;

- Minimum list of services and facilities necessary to enable a recipient to operate a business transferred to it under article 65(5), pubblicato il 20 maggio 2015;

- Technical advice on the delegated acts on critical functions and core business lines, pubblicato il 6 marzo 2015;

- $\quad$ RTS on the content of resolution plans and the assessment of resolvability, further specifying the contents of resolution plans for institutions and group and on the matters and criteria which resolution authorities should apply in the assessment of resolvability, pubblicato a marzo 2016;

- Implementing Technical Standards (ITS) on procedures, forms and templates for the provision of information for resolution plans under Article 11(3) of Directive 2014/59/EU of the European Parliament and the Council, pubblicato a giugno 2015. 


\title{
La contabilizzazione degli strumenti finanziari derivati nelle imprese di tipo non-financial alla luce del Decreto Legislativo 139/2015 e del principio contabile OIC 32
}

di Mattia Fabbri ${ }^{1}$ e Pier Giuseppe Giribone ${ }^{2} 3$

\begin{abstract}
The Legislative Decree no. 139 in August 2015 enables the recognition of derivatives in the financial statements of Italian entities adopting Italian Generally Accepted Accounting Principles (ITA GAAP). While in the financial statements for previous years derivatives were only disclosed in notes, according to new established rules, they are now measured at fair value.

A non-financial company can use derivatives for speculative purposes or for hedging risks. This poses interesting challenges related to the rationale inside the derivatives detention. Following Art. 2426 of Italian Civil Code, when a company holds derivatives for a cash flow hedge, changes (both positive and negative) in derivatives fair value must be recognized in a proper equity reserve. Indeed, if the derivative is held for speculative purposes, or even if it is not possible to prove its hedging vocation, the change directly affects the income statement. The recognition of the derivative to hedge the exposure to variability in cash flow or changes in fair value must comply with the set of characteristics established by the new Italian Accounting Standard OIC 32, which has been issued in 2016 and recently updated in December 2017 in the efforts to harmonize national and international accounting standards for derivatives.

Generally, small and medium-sized enterprises have inadequate resources in determining the value of derivatives that are traded over-the-counter (OTC). This is one of the reasons why many non-financial companies continue to refrain from trading some derivative financial instruments such as options in the OTC market. In fact, measuring the fair value of OTC options requires both specific knowledge of financial and statistical analysis methods and rather expensive software. However, bearing in mind the growing competitiveness of markets wherein companies operate today and the new disclosure obligations established by current legislation, all Italian non-financial companies will need to develop or improve their risk management practices. Only in this way they will be able to price complex derivatives (such as OTC options) and measure the efficiency in hedging strategies using those instruments.
\end{abstract}

\section{Key Words:}

Non-financial companies, recognition of speculative or hedging strategies, disclosures and accounting practices for financial derivatives, fair value in derivatives valuation and risk management, OTC markets, Directive 2013/34/UE, Legislative Decree n. 139/2015, Italian Accounting Standard OIC 32 (Derivatives), International Accounting Standard IFRS 13 (Fair Value Measurement).

\section{Introduzione}

Una caratteristica innegabile degli ultimi decenni è la maggior volatilità dei tassi di cambio, dei tassi di interesse e dei prezzi di mercato dei titoli e delle commodity rispetto ai decenni precedenti. Le fluttuazioni dei prezzi nei mercati finanziari possono avere effetti significativi sul patrimonio delle imprese. Variazioni su larga scala dei tassi di cambio possono indurre cambiamenti nella struttura competitiva dei mercati, a tal punto da determinare l'uscita di imprese da mercati nei quali in precedenza detenevano rassicuranti quote di mercato. Per esempio, l'apprezzamento del dollaro statunitense nella prima metà degli anni ottanta ebbe come conseguenza una netta perdita di quote di mercato domestiche ed estere di imprese statunitensi come Caterpillar e Kodak [1]. Il fatto ancor più grave è che, molto spesso, le imprese si rendono conto dell'importanza di questi rischi solo dopo essere incorse in significative perdite e quando ormai è troppo tardi.

In precedenza, gli shareholder e gli stakeholder accettavano il fatto che sfavorevoli e imprevedibili variazioni dei prezzi potessero incidere sul risultato finanziario dell'impresa. Oggigiorno, invece, essi si aspettano sempre più che il management sia in grado di identificare e gestire l'esposizione dell'impresa ai rischi di mercato. Tale compito è tipicamente affidato alle funzioni aziendali di risk management, che possono fare conto su una varietà di strumenti finanziari derivati al fine di trasferire, entro certi limiti e a fronte di un costo, tali rischi a soggetti terzi. Ciò nonostante, nella prima parte del presente articolo si vedrà come, alla luce delle statistiche pubblicate sull'attività di scambio nei mercati over-the-counter (di seguito OTC), la maggior parte delle imprese di tipo non-financial assumano decisioni finanziarie poco coerenti con gli scenari che si vanno configurando. Ad esempio, dopo la crisi economico-finanziaria ed i successivi anni di politiche monetarie espansive, risulta che molte imprese continuino ad astenersi dall'affacciarsi sul mercato degli strumenti finanziari derivati (in particolare di quelli più complessi come per esempio le opzioni) proprio quando, invece, le prospettive di un futuro cambiamento di strategia della Banca Centrale Europea parrebbero suggerire un loro prudente utilizzo nella gestione del rischio di tasso d'interesse e di cambio.

${ }^{1}$ Esperto Contabile iscritto all'Ordine dei Dottori Commercialisti e degli Esperti Contabili di Genova

${ }^{2}$ Università di Genova - Dipartimento di Economia (DIEC) e Banca CARIGE - Amministrazione Finanza

${ }^{3}$ Articolo sottoposto a doppio referaggio anonimo, pervenuto il 3/5/2018 e accettato il 03/07/2018. 
Nel prosieguo del presente articolo si illustreranno le modifiche al trattamento contabile dei derivati introdotte dal Decreto Legislativo n. 139 del 18 agosto 2015 (emanato in recepimento della direttiva 2013/34/UE) e dal nuovo principio contabile OIC 32 (Strumenti finanziari derivati), recentemente aggiornato dall'Organismo Italiano di Contabilità a fronte degli emendamenti pubblicati il 29 dicembre 2017. Come si vedrà, le nuove norme in tema di redazione del bilancio d'esercizio apportano un significativo contributo per migliorare la disclosure tecnico contabile dei contratti derivati sottoscritti da imprese di tipo non-financial che redigono il bilancio secondo i principi contabili nazionali. L'introduzione del fair value come criterio di base nella misurazione e rilevazione degli strumenti finanziari derivati è una novità nel nostro ordinamento che elimina molte delle precedenti differenze rispetto agli IAS/IFRS, ma al contempo comporta la necessità per tutte le imprese (anche per quelle di piccole e medie dimensioni come si vedrà nel prosieguo) di ampliare le proprie competenze per essere in grado di valutare correttamente tali strumenti finanziari e di verificare l'effettiva copertura di un derivato, anche in via quantitativa.

\section{Il mercato dei derivati corporate e gli strumenti maggiormente utilizzati dalle imprese non-financial}

Se da un lato le imprese utilizzano i derivati da molti anni, dall'altro si conosce ancora poco riguardo la loro diffusione e modalità di utilizzo nell'ambito delle imprese non-financial, in quanto, come si illustrerà nel prosieguo del presente articolo, solo recentemente è stata introdotta un'informativa contabile obbligatoria. Ciononostante, sono stati condotti negli anni numerosi studi, per lo più sotto forma di questionari e di survey, attraverso i quali si è in grado di comprendere quali siano le tecniche di risk management utilizzate in ambito corporate e, in particolare, quali siano i rischi da cui le imprese non-financial decidano di coprirsi e gli strumenti finanziari da esse maggiormente utilizzati per tale scopo [2]. Sebbene l'utilizzo dei derivati sia maggiormente diffuso tra le imprese più grandi in quanto la complessità della gestione di tali strumenti richiede notevoli conoscenze in campo finanziario e presenta rilevanti economie di scala, sulla base delle informazioni della Centrale dei rischi si rileva che il fenomeno risulti significativamente diffuso anche tra le imprese di medie e piccole dimensioni [3].

Un'ulteriore difficoltà che si incontra nel voler descrivere un quadro generale riguardo all'utilizzo del mercato dei derivati da parte delle imprese non-financial è determinata dal fatto che gli strumenti derivati utilizzati da queste imprese per finalità di copertura appartengono per lo più alla categoria dei derivati OTC, per i quali è piuttosto difficile arrivare ad una stima esatta dell'ammontare in gioco.

Tutto ciò premesso, si riportano di seguito alcune statistiche sia a livello internazionale, sia a livello nazionale riguardo i mercati regolamentati e OTC dei contratti di opzione. A livello internazionale, la Bank for International Settlements (di seguito BIS), in collaborazione con le banche centrali e le autorità monetarie di tutto il mondo, compila e diffonde varie serie di dati sull'attività bancaria e finanziaria internazionale. Fra tutte le informazioni elaborate, la BIS raccoglie e pubblica periodicamente statistiche sui derivati scambiati sia su mercati regolamentati, sia OTC. In particolare, la BIS mette insieme e pubblica tre diverse tipologie di statistiche che riguardano il mercato dei derivati: una trimestrale sui derivati negoziati su mercati organizzati, una semestrale sulle posizioni aperte nei mercati dei derivati OTC e una triennale sull'attività dei mercati di scambio di derivati OTC e di derivati su valute estere.

Le statistiche sui derivati della BIS riguardano il valore di mercato lordo (turnover) e le posizioni aperte (open interest) di contratti derivati su tassi di cambio e tassi di interesse. Le statistiche sono ricavate dai dati provenienti da 52 banche centrali, le quali a loro volta raccolgono i dati da banche e altri venditori della loro giurisdizione. La BIS non pubblica statistiche per i contratti azionari, di merci o di credito, né per i derivati che si riferiscono a strumenti sottostanti non standard (ad esempio inflazione, meteo o contratti energetici). Inoltre, i dati sul volume delle negoziazioni sono riportati dagli sportelli di vendita dei rivenditori segnalanti (sales desks), indipendentemente dal luogo in cui è registrata una transazione e sono segnalati su base non consolidata, ossia comprendendo le negoziazioni tra entità correlate che fanno parte dello stesso gruppo.

Tra tutte le indagini, la BIS Triennial Central Bank Survey è la più completa fonte di informazione sulla dimensione e sulla struttura dei mercati globali di strumenti derivati. L'ultima indagine è quella effettuata nel 2016, dalla quale emerge che la negoziazione con clienti non-financial, come aziende e governi, rappresenta solo il $7 \%$ del volume delle negoziazioni dei contratti derivati su cambi (in tendenza con quanto rilevato nei precedenti sondaggi) e 1'8\% di quello dei derivati su tassi di interesse OTC (in leggero rialzo rispetto ai dati precedenti) [4]. Inoltre, la survey fornisce utili informazioni riguardo alle diverse tipologie di strumenti derivati maggiormente negoziati a livello globale. In particolare, il volume di scambio delle opzioni e di altri strumenti finanziari comunque scomponibili in opzioni rappresenta circa il 5\% sul mercato dei cambi (il volume degli scambi di opzioni FX è sceso a 254 miliardi di dollari nel 2016, il 24\% in meno rispetto al 2013 ) e il 6\% sul mercato dei tassi di interesse, quest'ultimo in gran parte guidato dagli interest rate swap (il volume delle negoziazioni degli swap è aumentato del 33\% dal 2013, raggiungendo 1,9 trilioni di dollari nel 2016 e portando la sua quota di incidenza sul volume totale delle negoziazioni dei derivati su tassi di interesse OTC al 69\%).

In Figura 1 si riportano le medie giornaliere del volume delle negoziazioni OTC avvenute nel mese di aprile 2016 dei contratti derivati con sottostante valuta straniera, distinte per tipologia di strumento e controparte contrattuale. Come si può vedere le opzioni costituiscono una piccola parte dei contratti derivati su valuta straniera, in particolare la loro incidenza diminuisce sensibilmente quando la controparte è un'impresa non-financial. In tal caso, infatti, risulta che solo lo $0,6 \%$ degli strumenti derivati con sottostante valuta esterna siano costituiti da opzioni utilizzate dalle imprese non-financial per coprirsi dal rischio cambio. Da tale indagine emerge che per coprirsi dal rischio cambio le imprese preferiscono negoziare strumenti derivati diversi dalle opzioni, come forward, swap su valute estere o transazioni spot. Ciononostante, la quota del volume delle transazioni di opzioni su valuta esterna riferibili ad istituzioni non-financial, se considerata in termini assoluti, non può essere ritenuta trascurabile. Infatti, la media giornaliera di tali transazioni risulta essere di 30 miliardi di dollari e, come si vedrà tra 
poco, tale valore è più di tre volte superiore a quello corrispondente ai contratti di opzione su tassi di interesse in cui una controparte sia sempre un'impresa di tipo non-financial.

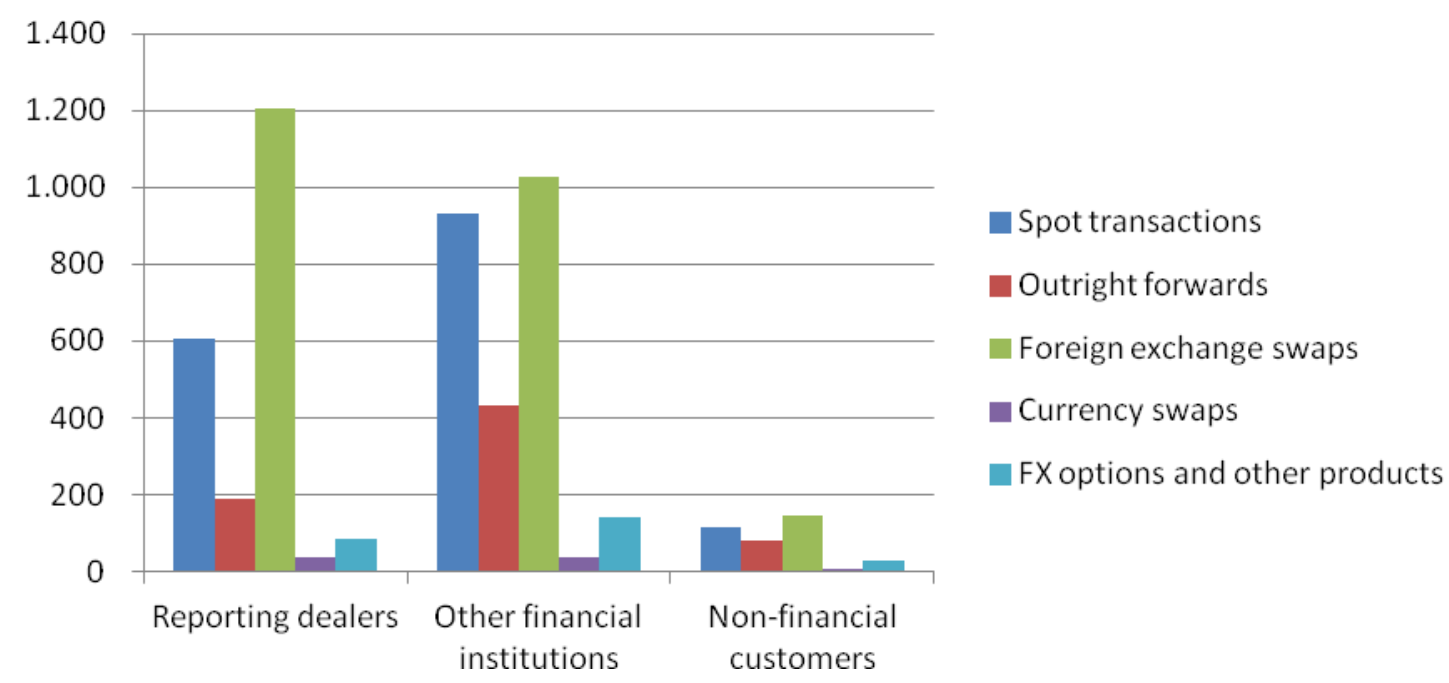

Figura 1. Volume medio giornaliero negoziato sui mercati globali ad aprile 2016 di derivati su valuta per tipologia e controparte (importi in miliardi di dollari)

In Figura 2 si riportano le medie giornaliere del volume delle negoziazioni OTC avvenute a livello globale nel mese di aprile 2016 dei contratti derivati che hanno come sottostante tassi di interesse. Anche qui si vede come in generale la compravendita di derivati su tassi di interesse e in particolare quella delle opzioni sui tassi di interesse è da attribuirsi per la maggior parte all'attività delle imprese finanziarie come banche, assicurazioni ed altre imprese finanziarie. Il volume medio giornaliero delle opzioni su tassi di interesse che abbiano come controparte un'istituzione non finanziaria è stimata in circa 9 miliardi di dollari, corrispondente soltanto allo $0,3 \%$ del volume complessivo delle negoziazioni di derivati che abbiano come sottostante tassi di interesse. Dalla figura emerge che le imprese di tipo non-financial, al fine di coprirsi dal rischio tasso d'interesse, utilizzano altre tipologie di contratti derivati, in particolare esse preferiscono rivolgersi al mercato dei contratti swap piuttosto che assumere posizioni contratti di opzione o forward.

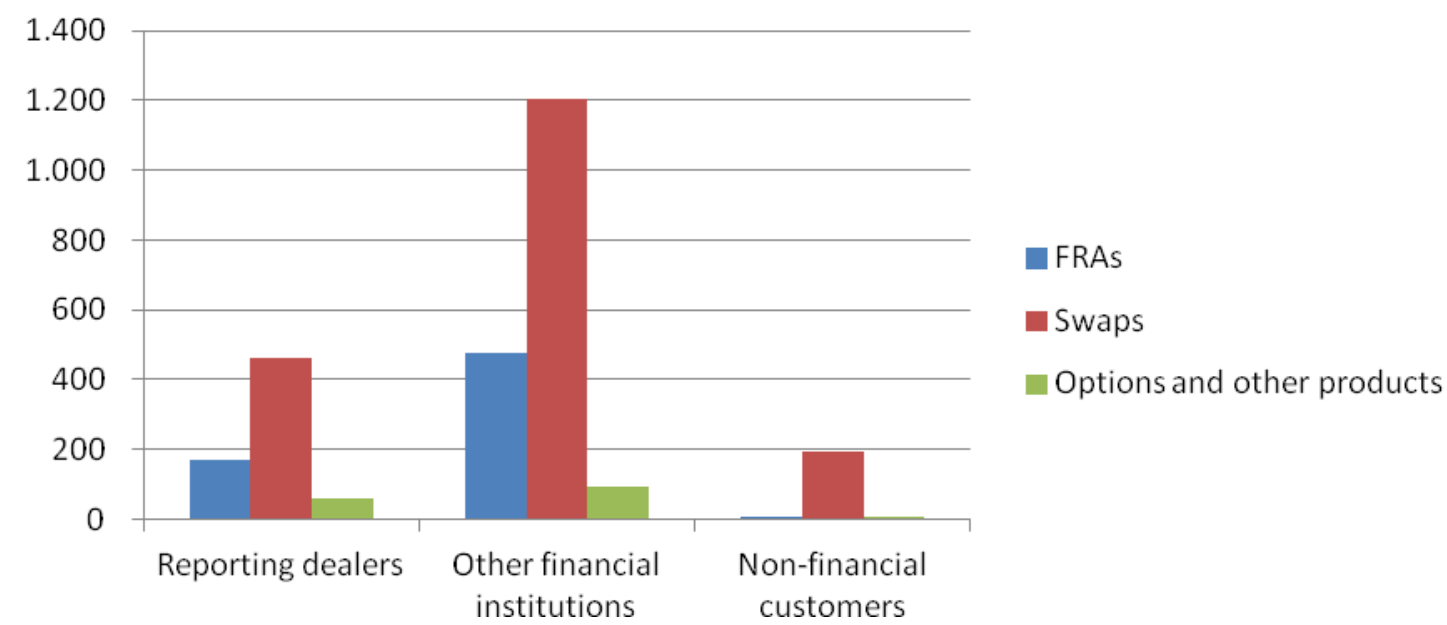

Figura 2. Volume medio giornaliero negoziato sui mercati globali ad aprile 2016 di derivati su tassi di interesse per tipologia e controparte (importi in miliardi di dollari)

A livello nazionale, la Banca d'Italia, nell'ambito dell'iniziativa del Committee on the Global Financial System (di seguito CGFS), comunica periodicamente le informazioni relative alle posizioni in derivati OTC per un campione composto dai sei maggiori gruppi bancari italiani (al campione considerato fa capo oltre il 90 per cento delle operazioni in derivati finanziari e creditizi). L'indagine è effettuata per iniziativa del CGFS e prevede, a livello globale, la rilevazione semestrale da parte della BIS di statistiche sui derivati OTC presso un campione di banche e intermediari finanziari maggiormente operativi nel comparto. Oggetto della rilevazione sono il valore nozionale e il valore lordo di mercato dei derivati OTC su tassi di cambio, tassi di interesse, azioni, indici azionari, commodity e credit default swap. A titolo di confronto con il mercato globale, si riporta di seguito una sintesi delle informazioni diffuse dalla Banca d'Italia relativamente alle posizioni in essere a fine dicembre 2016 [5]. In Figura 3 si vede come il mercato a livello nazionale, ancor più che a livello globale, sia dominato dalle istituzioni finanziarie. In Italia, infatti, sull'importo nominale totale degli strumenti derivati OTC in essere a dicembre 2016 (pari a 8.398 miliardi di dollari), solo il 4,3\% delle negoziazioni (corrispondente al nozionale di 360 miliardi di dollari) 
avviene con controparti di tipo non-financial (di cui 1'1,5\% hanno come sottostante tassi di cambio, il 2,7\% tassi d'interesse e lo $0,1 \%$ titoli azionari).

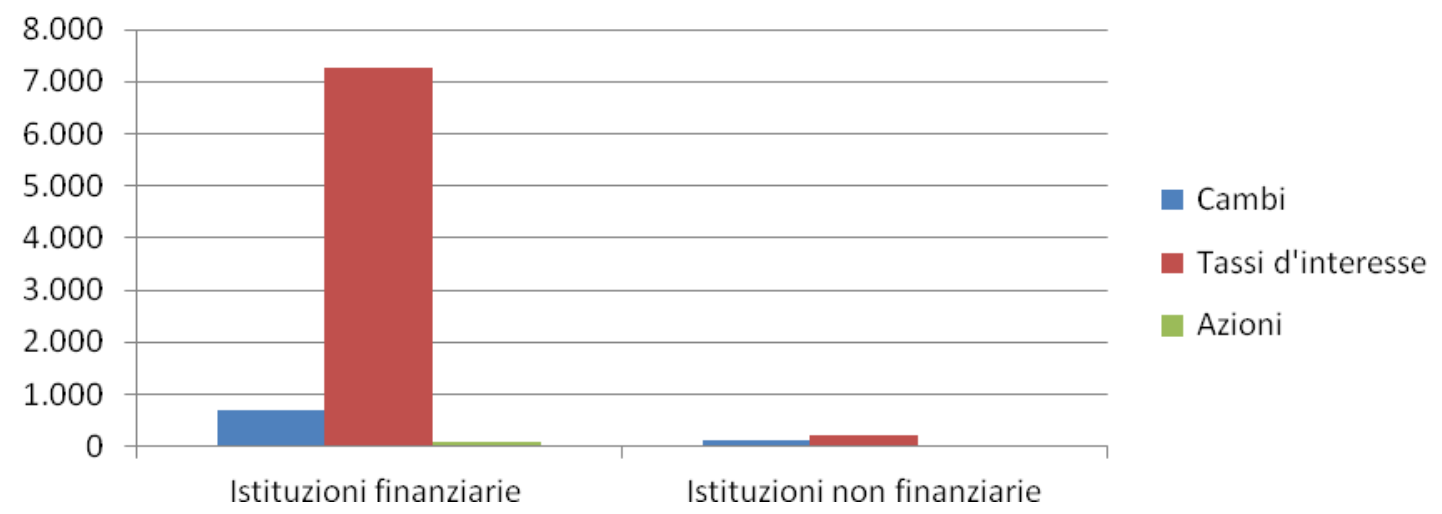

Figura 3. Contratti derivati per categoria di rischio e controparte negoziati OTC sul mercato italiano in essere a dicembre 2016 (importi in miliardi di dollari)

Inoltre, se si considerano i due prodotti maggiormente negoziati tra imprese non-financial, ossia i derivati su tassi di interesse e quelli su tassi di cambio, si evidenzia a livello nazionale un'incidenza più alta, rispetto quella globale, dell'uso delle opzioni rispetto ad altri strumenti derivati. In particolare, come si vede in Figura 4, tra tutti gli strumenti derivati negoziati OTC, le opzioni costituiscono il 9,4\% degli strumenti destinanti alla copertura del rischio tasso di interesse (pari ad un nozionale di 430 miliardi di dollari) e ben il 12,4\% degli strumenti derivati su valuta estera (pari ad un nozionale di 202 miliardi di dollari).

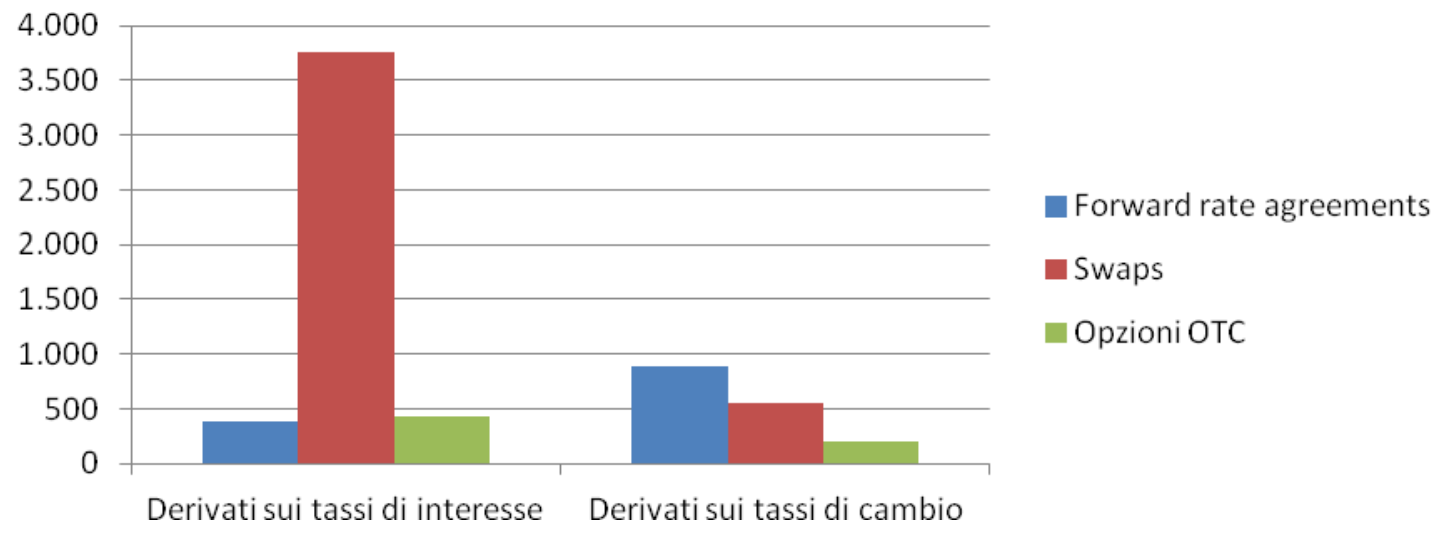

Figura 4. Contratti derivati per strumento e categoria di rischio negoziati OTC sul mercato italiano in essere a dicembre 2016 (importi in miliardi di dollari)

Dal quadro che si è appena presentato emerge che le imprese di tipo non-financial utilizzano i contratti derivati tipicamente per coprirsi dal rischio valuta e dal rischio tasso di interesse, ma al contempo emerge che l'utilizzo in generale degli strumenti finanziari derivati e in particolare di quelli più complessi come per esempio le opzioni da parte delle imprese non-financial è ancora modesto.

Con il recente comunicato stampa del 12 giugno 2018, la Banca d'Italia ha diffuso le informazioni relative alle posizioni in derivati OTC a fine dicembre 2017 [6]. In Tabella 1 e in Tabella 2 si riportano i dati di sintesi rispettivamente della rilevazione effettuata dalla Banca d'Italia a fine dicembre 2016 e di quella più recente effettuata a fine dicembre 2017. Dal confronto emerge che qualcosa sta cambiando nel comportamento delle imprese. In particolare, si rileva un aumento dell'ammontare delle posizioni in derivati in cui la controparte è un'istituzione non finanziaria, specialmente per la copertura del rischio tasso d'interesse.

\begin{tabular}{|l|r|r|r|r|r|r|}
\hline Categoria di rischio & \multicolumn{2}{|c|}{ Istituzioni finanziarie } & \multicolumn{2}{c|}{ Istituzioni non finanziarie } & \multicolumn{2}{c|}{ TOTALE } \\
\hline & Importo & Composizione & Importo & Composizione & Importo & Composizione \\
\hline Cambi & 688,4 & 84,1 & 130,0 & 15,9 & 818,4 & 9,7 \\
\hline Tassi d'interesse & $7.252,5$ & 97,0 & 226,0 & 3,0 & $7.478,5$ & 89,1 \\
\hline Azioni & 96,5 & 95,6 & 4,4 & 4,4 & 100,9 & 1,2 \\
\hline TOTALE & $8.037,4$ & 95,7 & 360,4 & 4,3 & $8.397,8$ & 100,0 \\
\hline
\end{tabular}

Tabella 1. Contratti derivati per controparte e categoria di rischio negoziati OTC sul mercato italiano in essere a dicembre 2016 (importi in miliardi di dollari e percentuali) [5] 


\begin{tabular}{|l|r|r|r|r|r|r|}
\hline \hline Categoria di rischio & \multicolumn{2}{|c|}{ Istituzioni finanziarie } & \multicolumn{2}{c|}{ Istituzioni non finanziarie } & \multicolumn{2}{c|}{ TOTALE } \\
\hline & Importo & Composizione & Importo & Composizione & Importo & Composizione \\
\hline Cambi & 772,9 & 83,7 & 150,5 & 16,3 & 923,4 & 13,8 \\
\hline Tassi d'interesse & $5.344,9$ & 94,3 & 324,7 & 5,7 & $5.669,6$ & 84,7 \\
\hline Azioni & 96,3 & 95,2 & 4,9 & 4,8 & 101,2 & 1,5 \\
\hline TOTALE & $6.214,1$ & 92,8 & 480,1 & 7,2 & $6.694,2$ & 100,0 \\
\hline
\end{tabular}

Tabella 2. Contratti derivati per controparte e categoria di rischio negoziati OTC sul mercato italiano in essere a dicembre 2017 (importi in miliardi di dollari e percentuali) [6]

\section{La determinazione del fair value degli strumenti finanziari derivati alla luce della direttiva 2013/34/UE}

Il recepimento della direttiva 2013/34/UE "relativa ai bilanci d'esercizio, ai bilanci consolidati e alle relative relazioni di talune tipologie di imprese, recante modifica della Direttiva 2006/43/CE del Parlamento europeo e del Consiglio e abrogazione delle direttive 78/660/CEE e 83/349/CEE del Consiglio" (nel seguito nuova direttiva contabile) ha comportato un'importante innovazione nella prassi contabile nazionale, introducendo, tra l'altro, l'obbligo per tutte le imprese, incluse anche le imprese che redigono il bilancio in forma abbreviata ai sensi dell'articolo 2435 bis del Codice Civile, di adottare una più trasparente e completa informativa di bilancio in tema di strumenti finanziari derivati.

In tema di strumenti finanziari derivati la nuova direttiva contabile, in conformità con l'obiettivo di aumentare la chiarezza e la comparabilità dei bilanci, prevede che "gli Stati membri autorizzino o prescrivano, per tutte le imprese o per talune categorie di imprese, la valutazione al fair value degli strumenti finanziari, compresi gli strumenti finanziari derivati”, in deroga ai principi generali di valutazione, i quali prevedono invece che le voci rilevate nel bilancio siano valutate secondo il principio del prezzo di acquisto o del costo di produzione.

Il legislatore comunitario precisa quale debba essere la modalità di determinazione del fair value di uno strumento derivato, prevedendo che il valore sia determinato con riferimento a uno dei seguenti valori:

a) nel caso di strumenti finanziari per i quali sia possibile individuare facilmente un mercato attendibile, al valore di mercato; qualora il valore di mercato non sia facilmente individuabile per un dato strumento, ma possa essere individuato per i suoi componenti o per uno strumento analogo, il valore di mercato può essere derivato da quello dei componenti o dello strumento analogo;

b) nel caso di strumenti finanziari per i quali non sia possibile individuare facilmente un mercato attendibile, al valore che risulta da modelli e tecniche di valutazione generalmente accettati, purché questi modelli e tecniche di valutazione assicurino una ragionevole approssimazione al valore di mercato.

Al riguardo si sottolinea come il legislatore comunitario, pur non facendo esplicito richiamo ai principi contabili internazionali, si ispiri alle norme in tema di gerarchia dei livelli di fair value contenute nell'IFRS 13 per definire quali siano le informazioni utili alla determinazione del valore degli strumenti finanziari, inclusi quelli derivati. In particolare, per aumentare la coerenza e la comparabilità delle valutazioni del fair value e delle relative informazioni integrative, l'IFRS 13 stabilisce una gerarchia del fair value che classifica in tre livelli gli input delle tecniche di valutazione adottate per la determinazione del fair value. La gerarchia del fair value stabilita dall'IFRS 13, così come introdotta dalla nuova direttiva contabile, è dunque la seguente:

livello 1) prezzi di mercato;

livello 2) input osservati sul mercato;

livello 3) input non osservati sul mercato.

\section{L'iscrizione a bilancio degli strumenti finanziari derivati alla luce del Decreto Legislativo n. 139/2015}

L'Italia ha recepito nel proprio ordinamento interno la nuova direttiva contabile con l'entrata in vigore del D.Lgs n. 139 del 18 agosto 2015 (di seguito Decreto Bilanci), che ha riformato le precedenti regole civilistiche in materia di bilancio, introducendo norme interne del tutto nuove per la nostra prassi contabile tra cui la contabilizzazione degli strumenti finanziari derivati. La riforma contabile, infatti, ha reso obbligatoria la contabilizzazione dei derivati a partire dai bilanci 2016, introducendo il nuovo numero 11 bis) del primo comma dell'articolo 2426 del Codice Civile, il quale prevede, ai sensi della nuova direttiva contabile, che gli strumenti finanziari derivati, anche se incorporati in altri strumenti finanziari, siano iscritti al fair value.

Fino ai bilanci 2015 i derivati di copertura e quelli messi in atto per arbitrare sull'andamento di un prezzo o di un tasso (cosiddetti speculativi) trovavano rappresentazione in nota integrativa, ai sensi dell'articolo 2427 bis del Codice Civile, a meno che rappresentassero una passività probabile, nel qual caso sarebbero stati opportunamente rilevati tra $\mathrm{i}$ fondi per rischi ed oneri. Invece, con decorrenza 1 gennaio 2016, ai sensi del nuovo disposto del citato articolo così come modificato dal Decreto Bilanci, un derivato deve essere iscritto tra le attività o le passività dello stato patrimoniale, a seconda che il suo fair value sia positivo o negativo.

A tale scopo sono state operate le seguenti modifiche all'articolo 2424 del Codice Civile, il quale stabilisce il contenuto dello schema di stato patrimoniale:

- alla voce dell'attivo B.III "Immobilizzazioni finanziarie" il punto 4) "Strumenti finanziari derivati attivi", ha sostituito il precedente "Azioni proprie"; 
- la voce dell'attivo C.III "Attività finanziarie che non costituiscono immobilizzazioni" il punto 5) "Strumenti finanziari derivati attivi", ha sostituito il precedente "Azioni proprie";

- alla voce del passivo B "Fondi per rischi ed oneri" il punto 3) "Strumenti finanziari derivati passivi", ha sostituito il precedente "Altri [fondi per rischi ed oneri]" che ritroviamo invece ora al successivo punto 4).

La disposizione introdotta, ispirata alla migliore prassi contabile internazionale, prevede un generale obbligo di rilevazione degli strumenti derivati al fair value. La norma, infatti, chiarisce che l'obbligo di valutazione al fair value debba essere esteso anche alle circostanze nelle quali lo strumento derivato è inglobato in un altro titolo, riferendosi ai cosiddetti "derivati impliciti" (si veda a tal proposito il successivo paragrafo 5.1).

Nel prosieguo della norma contenuta nel sopracitato numero 11 bis) è stabilito che le variazioni del fair value siano imputate al conto economico e, a tal fine, l'articolo 2425 del Codice Civile, che stabilisce il contenuto dello schema del conto economico, è stato anche'esso modificato per recepire gli effetti sulle voci di bilancio derivanti dalla nuova disciplina sui derivati, aggiungendo:

- alla voce D.18 "Rivalutazioni", la lettera d) "di strumenti finanziari derivati";

- alla voce D.19 "Svalutazioni" la lettera d) "di strumenti finanziari derivati".

Detto ciò, la norma, coerentemente a quanto previsto dalla nuova direttiva contabile, prevede un'eccezione alla rilevazione in conto economico, disponendo che, qualora lo strumento copra il rischio di variazione dei flussi finanziari attesi di un altro strumento finanziario o di un'operazione programmata, le variazioni di fair value siano imputate direttamente ad una riserva positiva o negativa di patrimonio netto. A tal scopo l'articolo 2424 del Codice Civile è stato altresì modificato, aggiungendo al passivo dello stato patrimoniale la voce A.VII "Riserva per operazioni di copertura di flussi finanziari attesi".

Nel caso di strumenti derivati utilizzati ai fini di copertura dei rischi, la norma prevede un regime differenziato a seconda che la copertura si riferisca al fair value di elementi presenti nel bilancio (fair value hedge) oppure a flussi finanziari o operazioni di futura manifestazione (cash flow hedge). Ferma restando la valutazione al fair value del derivato, nel primo caso la norma richiede di valutare l'elemento oggetto di copertura evidenziando le variazioni di valore relative al rischio coperto a conto economico; nel secondo caso, in assenza di elementi da valutare in bilancio, in quanto la copertura si riferisce a fenomeni di futura manifestazione, gli effetti della valutazione al fair value sono rilevati direttamente a patrimonio netto, in una riserva che può essere positiva o negativa [8]. Di seguito la norma dispone che tale riserva sia imputata al conto economico nella misura e nei tempi corrispondenti al verificarsi o al modificarsi dei flussi di cassa dello strumento coperto o al verificarsi dell'operazione oggetto di copertura. Dunque, solo una volta manifestatisi gli effetti dell'operazione a cui si è inteso dare copertura, gli utili o le perdite maturati sullo strumento derivato sono rilevati nel conto economico così da sterilizzare le oscillazioni di valore sull'elemento coperto oggetto di copertura.

In Figura 5 si riportano graficamente le possibili modalità di rilevazione in bilancio delle variazioni di fair value per i derivati non di copertura e per quelli di copertura.

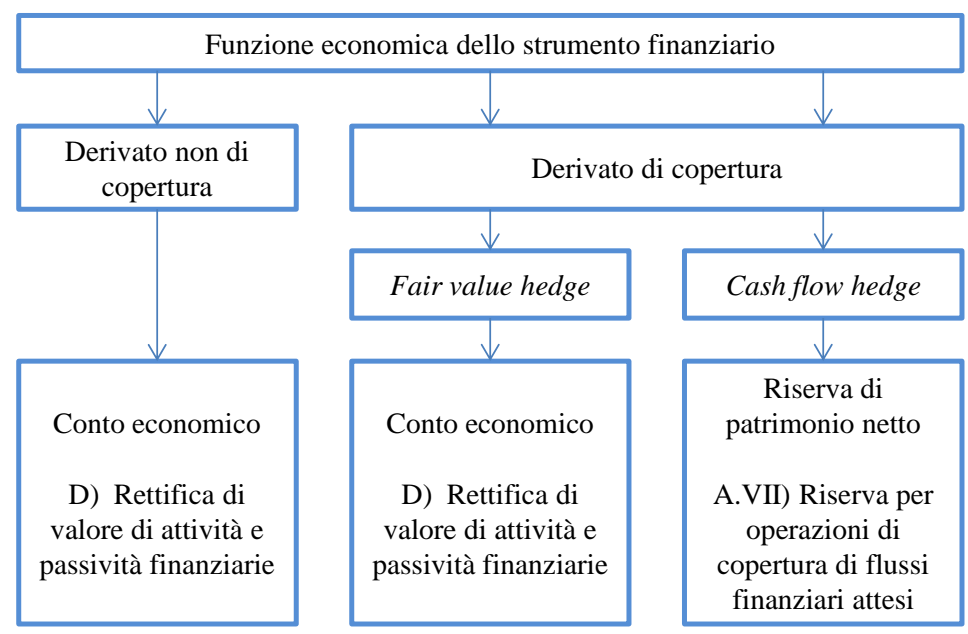

Figura 5. Modalità di rilevazione a bilancio delle le variazioni del fair value degli strumenti finanziari derivati ai sensi del nuovo disposto dell'articolo 2424 del Codice Civile [7]

Quindi, la contabilizzazione in bilancio degli strumenti finanziari derivati presuppone, come si è visto, la loro classificazione in una delle due categorie previste dalla norma. A questi fini, è necessario individuare l'esistenza o meno di una relazione di copertura tra lo strumento finanziario derivato e il suo sottostante. Per maggiori dettagli in merito alla contabilizzazione dei derivati nelle operazioni di copertura si rimanda al successivo paragrafo 5.2 del presente articolo, in particolare si rimanda al sottoparagrafo 5.2.1 per le operazioni di copertura di attività o passività iscritte in bilancio (fair value hedge) e al sottoparagrafo 5.2.2. per le operazioni di copertura dei flussi finanziari attesi (cash flow hedge). Nel caso di derivati detenuti per finalità speculative o nei casi in cui non sia possibile o non si voglia dimostrare una relazione di copertura, le variazioni di valore del derivato devono essere imputate sempre a conto economico.

Gli elementi oggetto di copertura contro il rischio di variazioni dei tassi di interesse o dei tassi di cambio o dei prezzi di mercato o contro il rischio di credito devono essere valutati simmetricamente allo strumento derivato di copertura. Ciò significa che la contabilizzazione dell'elemento coperto segue quella del derivato, ossia lo strumento di copertura. In altre 
parole, le variazioni di fair value dell'elemento coperto attribuibili al rischio oggetto di copertura devono essere contabilizzate a conto economico, compensando in tal modo le variazioni di fair value del derivato, anch'esse rilevate a conto economico. Un passaggio molto importante della nuova norma riguarda la relazione di copertura che deve esistere tra l'elemento coperto e lo strumento di copertura. A tal proposito la norma civilistica, disponendo che si debba considerare sussistente la copertura in presenza, fin dall'inizio, di stretta e documentata correlazione tra le caratteristiche dello strumento o dell'operazione oggetto di copertura e quelle dello strumento di copertura, appare non sufficientemente esaustiva, rinviando di fatto implicitamente ai principi contabili nazionali per la necessaria applicazione pratica della stessa (si veda a tal proposito il successivo paragrafo 5.3).

Tale criterio di valutazione per gli strumenti derivati, pur comportando una rappresentazione veritiera e corretta delle operazioni medesime, potrebbe in specifiche circostanze far venire meno il principio della prudenza, derogando al presupposto generale che prevede la rilevazione a bilancio esclusivamente per gli utili realizzati alla data di chiusura del bilancio. Per tenere conto di tale aspetto, la norma in commento dispone che non siano distribuibili gli utili che derivano dalla valutazione al fair value degli strumenti finanziari derivati non utilizzati o non necessari per la copertura. Con tale disposizione il legislatore nazionale previene, vietandole, azioni potenzialmente lesive dell'integrità del capitale. Dunque, nel caso di strumenti finanziari non inquadrati in operazioni di copertura, gli eventuali utili derivanti dalla valutazione al fair value sono accantonati in una riserva non distribuibile; tale obbligo non sussiste nel caso invece di utili derivanti dalla valutazione di strumenti derivati di copertura, a condizione che la copertura si riferisca ad elementi presenti nel bilancio e valutati simmetricamente allo strumento derivato di copertura e che non vi siano componenti inefficaci della copertura.

Le riserve di patrimonio che si generano per effetto della valutazione al fair value di derivati utilizzati a copertura dei flussi finanziari di un altro strumento finanziario o di operazioni di futura manifestazione, non trovando corrispondenza in bilancio con utili o perdite di segno opposto fino a che non si manifestano i flussi o le operazioni oggetto di copertura, non sono considerate nel computo del patrimonio netto per le finalità di cui agli articoli 2412, 2433, 2442, 2446 e 2447 del Codice Civile e, se positive, non sono disponibili e non sono utilizzabili a copertura delle perdite [8]. Con tale norma il legislatore dispone che tali riserve, derivanti dalla valutazione al fair value di derivati di copertura, qualora siano negative, non debbano essere considerate nel computo del patrimonio netto per la determinazione dei limiti all'emissione di obbligazioni (art. 2412), della distribuzione degli utili ai soci (art. 2433), del passaggio da riserve a capitale (art. 2442), per il conteggio della riduzione del capitale per perdite (art. 2446) e per il conteggio della riduzione del capitale sociale al di sotto del limite legale (art. 2447). Invece, qualora risultino positive, tali riserve devono essere classificate come indisponibili e, dunque, assoggettate non solo al vincolo di indistribuibilità già previsto per gli eventuali utili derivanti dalla valutazione al fair value di derivati non inquadrati in operazioni di copertura, ma al più rigido vincolo di indisponibilità. Per non lasciare dubbi in merito ad eventuali deroghe, anche se forse non sarebbe stato nemmeno necessario visto il tenore generale della norma, il legislatore specifica che tali riserve non sono altresì disponibili al fine della copertura delle perdite.

\section{La rilevazione, classificazione e valutazione degli strumenti finanziari derivati secondo l'OIC 32}

L'attività di aggiornamento dei principi contabili nazionali sulla base delle norme contenute nel Decreto Bilanci ha portato all'emanazione nel dicembre 2016 del nuovo principio contabile OIC 32 "Strumenti finanziari derivati" per disciplinare i criteri per la rilevazione, classificazione e valutazione dei derivati, nonché le informazioni da presentare in nota integrativa. Il documento OIC recepisce le novità introdotte con il suddetto decreto e sviluppa una robusta normativa secondaria che spazia dalla definizione di strumento derivato, ai criteri per individuare e scorporare i derivati impliciti, al calcolo del fair value (che diviene criterio unico di rilevazione), alla definizione dei derivati di copertura (sia di fair value che di flussi finanziari), alla verifica della relazione di copertura, alle regole di contabilizzazione e iscrizione in bilancio. Il documento OIC 32 sostituisce completamente, abrogandole, le disposizioni sugli strumenti finanziari derivati di cui all'OIC 3 (Le informazioni sugli strumenti finanziari da includere nella nota integrativa e nella relazione sulla gestione). Recentemente, con la definitiva approvazione degli emendamenti ai principi OIC avvenuta il 29 dicembre 2017, dopo un periodo di consultazione del documento in bozza, sono operative alcune novità di seguito illustrate che trovano applicazione ai bilanci con esercizio avente inizio a partire dal $1^{\circ}$ gennaio 2017 o da data successiva.

\subsection{Ambito di applicazione}

Sotto il profilo soggettivo, il nuovo principio contabile OIC 32 si applica alle società che redigono il bilancio in base alle disposizioni del Codice Civile, ivi incluse le imprese che redigono il bilancio in forma abbreviata tenute ad applicare la disciplina prevista dall'art. 2426, c.1 del Codice Civile. Restano dunque escluse dalla sua applicazione le società che redigono il bilancio secondo i principi contabili internazionali IAS/IFRS (le quali procederanno all'iscrizione e alla valutazione dei derivati in conformità ai principi contabili internazionali), le micro-imprese (che sono esonerate dall'applicazione della nuova disciplina introdotta in tema di strumenti finanziari derivati e sono definite dall'articolo 2435-ter del Codice Civile come quelle imprese che nel primo esercizio o per due esercizi consecutivi non superano due dei seguenti limiti: totale attivo pari a $175.000 €$; totale ricavi delle vendite e delle prestazioni pari a $350.000 €$; dipendenti occupati in media nell'esercizio pari a 5 unità) e, per esplicita disposizione di legge, le società assicurative (le quali applicheranno le disposizioni previste dal codice delle assicurazioni private).

Riguardo, invece, all'ambito di applicazione oggettivo, l'OIC 32 definisce un derivato uno strumento finanziario o un altro contratto che possieda le seguenti tre caratteristiche: 
a) il suo valore varia come conseguenza della variazione di un determinato tasso di interesse, prezzo di strumenti finanziari, prezzo di merci, tasso di cambio, indice di prezzo o di tasso, rating di credito o indice di credito o altra variabile, a condizione che, nel caso di una variabile non finanziaria, tale variabile non sia specifica di una delle controparti contrattuali (a volte chiamato il sottostante);

b) non richiede un investimento netto iniziale o richiede un investimento netto iniziale che sia minore di quanto sarebbe richiesto per altri tipi di contratti da cui ci si aspetterebbe una risposta simile a variazioni di fattori di mercato;

c) è regolato a data futura.

Detto ciò, l'OIC 32, per espressa disposizione, esclude comunque dall'ambito di applicazione dalla disciplina:

a) i contratti derivati aventi ad oggetto le azioni proprie della società;

b) i contratti derivati stipulati tra un acquirente e un venditore relativi agli strumenti di capitale oggetto di un'operazione straordinaria;

c) le opzioni di riscatto incluse nei contratti di leasing;

d) le relazioni di copertura in cui lo strumento di copertura è un'attività o passività finanziaria non derivata.

Dunque, la disciplina dei derivati non è limitata agli strumenti finanziari, bensì è applicabile a qualunque forma contrattuale (ad esempio a un contratto di acquisto di materie prime), a condizione che siano presenti le tre caratteristiche testé elencate, ovvero salvo quanto specificato dal comma 3 dell'art. 2426 del Codice Civile, nonché dai paragrafi 6-8 del principio OIC 32. In dettaglio, ai sensi dell'articolo 2426, comma 3, del Codice Civile rientrano nell'ambito di applicazione del principio OIC 32 anche quei contratti di acquisto e vendita di merci che conferiscono all'una o all'altra parte contraente il diritto di procedere alla liquidazione del contratto per contanti o mediante altri strumenti finanziari ad eccezione del caso in cui si verifichino contemporaneamente le seguenti condizioni:

a) il contratto sia stato concluso e sia mantenuto per soddisfare le esigenze di acquisto, vendita o di utilizzo merci;

b) il contratto sia destinato a tale scopo fin dalla sua conclusione;

c) si preveda che il contratto sia eseguito mediante consegna della merce.

In relazione a tali contratti, l'OIC 32 precisa che se la società segue una prassi di regolamento al netto sulla base di disponibilità liquide o mediante altri strumenti finanziari, oppure per prassi riceve la merce oggetto del contratto per poi rivenderla nel breve periodo dopo la consegna al fine di generare un utile dalle fluttuazioni a breve termine del prezzo o dal margine di profitto dell'operatore, non può invocare l'aspettativa della consegna della merce per non valutare il contratto come uno strumento finanziario derivato. Ad eccezione di quanto appena detto, però, il principio prevede che un'opzione (call o put) venduta, per l'acquisto o la vendita di merci, che dà diritto ad entrambe le parti di regolare il contratto al netto sulla base di disponibilità liquide o mediante altri strumenti finanziari oppure che ha come sottostante merce facilmente liquidabile è da contabilizzarsi come fosse uno strumento finanziario derivato, anche se nella prassi la società procede alla consegna fisica della merce.

Inoltre, il principio definisce un contratto ibrido un contratto composto da uno strumento finanziario derivato (derivato incorporato) e un contratto primario (contratto non derivato regolato a normali condizioni di mercato). Un contratto ibrido genera flussi finanziari che non avrebbero avuto luogo se non fosse stata presente la componente derivativa. Ad esempio un finanziamento che preveda il pagamento di interessi in base all'andamento dell'indice di prezzo dell'oro contiene una componente derivativa. In tale circostanza il contratto di finanziamento (contratto ibrido) si compone di un contratto di finanziamento a condizioni normali di mercato (contratto primario) e di uno swap (derivato incorporato).

Secondo il presente principio, un derivato incorporato deve essere separato dal contratto primario e contabilizzato come uno strumento finanziario derivato se, e soltanto se:

a) le caratteristiche economiche e i rischi del derivato incorporato non sono strettamente correlati alle caratteristiche economiche e ai rischi del contratto primario. In tal senso il principio chiarisce che sussiste una stretta correlazione nei casi in cui il contratto ibrido è stipulato secondo le prassi di mercato;

b) sono soddisfatti tutti gli elementi della definizione di strumento finanziario derivato testé elencati.

Si noti che un derivato incorporato deve essere separato dal contratto primario anche nei casi in cui quest'ultimo non abbia natura finanziaria (ad esempio nel caso di un contratto di acquisto di merci).

\subsection{La contabilizzazione dei derivati nelle operazioni di copertura}

Nell'impianto normativo che si è delineato, il legislatore nazionale ha scelto la tecnica giuridica del rinvio richiamando esplicitamente i principi contabili internazionali per la definizione di strumento finanziario, di strumento derivato e di fair value. Per tale ragione, l'OIC 32 riprende la definizione fornita dall'IFRS 13 e definisce il fair value il prezzo che si percepirebbe per la vendita di un'attività ovvero che si pagherebbe per il trasferimento di una passività in una regolare operazione tra operatori di mercato alla data di valutazione. Dunque, la valutazione del fair value di uno strumento finanziario derivato richiede ad una società di determinare:

a) lo strumento finanziario derivato oggetto della valutazione;

b) il mercato principale (o più vantaggioso) per lo strumento finanziario derivato;

c) le tecniche di valutazione appropriate per la valutazione che devono considerare:

i. $\quad$ il livello della gerarchia del fair value in cui sono classificati i parametri;

ii. le assunzioni che gli operatori di mercato utilizzerebbero per determinare il prezzo dello strumento finanziario derivato, incluse le assunzioni circa i rischi, presumendo che gli operatori di mercato agiscano per soddisfare nel modo migliore il proprio interesse economico. 
In quanto l'uso di derivati di copertura può incidere sulla modalità di contabilizzazione di tali strumenti, l'OIC dedica, similmente alle regole dello IASB, ampio spazio all'utilizzo dei derivati in operazioni di copertura. Il principio contabile ammette la contabilizzazione di operazioni di copertura solo per i seguenti rischi:

a) rischio di tasso d'interesse, ad esempio, di uno strumento di debito rilevato al costo ammortizzato;

b) rischio di cambio, ad esempio il rischio di cambio su un acquisto futuro altamente probabile in valuta estera;

c) rischio di prezzo, ad esempio di una merce in magazzino o di un titolo azionario detenuto dalla società;

d) rischio di credito (ad esclusione del rischio di credito proprio della società).

Ogni derivato di copertura deve essere riconducibile a un elemento coperto, che in linea generale può essere un'attività, una passività, un impegno irrevocabile, un'operazione programmata altamente probabile che (a) espone la società al rischio di variazioni nel fair value o nei flussi finanziari futuri e (b) è designato come coperto. Un impegno irrevocabile è un accordo vincolante per lo scambio di una quantità prestabilita di risorse ad un prezzo prestabilito ad una data o più date future prestabilite. Un'operazione programmata, invece, è un'operazione futura per la quale non vi è ancora un impegno irrevocabile. Per il chiarimento del significato di "altamente probabile" si rimanda al successivo paragrafo 5.3.

L'esistenza di una relazione di copertura implica che il valore dello strumento di copertura evolve, in genere, nella direzione opposta del valore dell'elemento coperto in conseguenza di uno stesso rischio, che è il rischio oggetto di copertura. L'efficacia della copertura è il livello a cui le variazioni nel fair value o nei flussi finanziari dell'elemento coperto, che sono attribuibili ad un rischio coperto, sono compensate dalle variazioni nel fair value o nei flussi finanziari dello strumento di copertura. L'inefficacia della copertura è il grado in cui le variazioni del fair value o dei flussi finanziari dello strumento di copertura superano o sono inferiori a quelle dell'elemento coperto. Il rapporto di copertura è il rapporto tra la quantità dello strumento di copertura e la quantità dell'elemento coperto in termini di peso relativo.

Detto ciò, le relazioni di copertura possono essere di due tipi:

a) copertura delle variazioni di fair value (fair value hedge): si applica nei casi in cui l'obiettivo della copertura è quello di limitare l'esposizione al rischio delle variazioni di fair value di attività, passività iscritte in bilancio o impegni irrevocabili, che in assenza di una copertura potrebbero influenzare il risultato d'esercizio;

b) copertura di flussi finanziari (cash flow hedge): si applica nei casi in cui l'obiettivo della copertura è quello di limitare l'esposizione al rischio di variabilità dei flussi finanziari attribuibili ad attività, passività iscritte in bilancio, ad impegni irrevocabili oppure operazioni programmate altamente probabili, che in assenza di una copertura potrebbero influenzare il risultato d'esercizio.

\subsubsection{Copertura di fair value (fair value hedge)}

La copertura di fair value può essere attivata quando l'elemento coperto è un'attività o una passività iscritta in bilancio oppure un impegno irrevocabile. È questo il caso di un portafoglio di titoli azionari detenuto dalla società con l'obiettivo di mantenerlo in portafoglio per un periodo di tempo determinato senza correre il rischio di un deprezzamento del valore di mercato. Un altro esempio di copertura del fair value è quello di un magazzino di materie prime che, per strategia aziendale, possono essere destinate sia alla produzione interna di prodotti finiti sia alla vendita a terzi. Anche in questo caso l'obiettivo della copertura non è quello di stabilizzare i flussi finanziari attesi, bensì di evitare un deprezzamento del valore del magazzino materie prime.

La copertura di fair value lungo la sua durata deve essere contabilizzata come segue:

a) lo strumento di copertura (cioè lo strumento finanziario derivato) deve essere valutato al fair value e quindi rilevato nello stato patrimoniale come un'attività o una passività;

b) l'elemento coperto, in deroga ai principi di riferimento, è valutato nei seguenti modi:

i. nel caso di un'attività o una passività iscritta in bilancio, il valore contabile è adeguato per tener conto della valutazione al fair value della componente relativa al rischio oggetto di copertura (l'adeguamento del valore contabile di un'attività avviene nei limiti del valore recuperabile);

ii. nel caso di un impegno irrevocabile, il fair value della componente relativa al rischio oggetto di copertura è iscritta nello stato patrimoniale come attività o passività nella voce di stato patrimoniale che sarà interessata dall'impegno irrevocabile al momento del suo realizzo.

In sostanza, quindi, l'elemento coperto sarà contabilizzato inizialmente secondo il criterio stabilito per la classe (per esempio il magazzino al minore tra costo e valore di mercato). Quindi, se le oscillazioni del suo fair value sono oggetto di copertura contabile, il suo valore andrà adeguato lungo la durata della copertura per le variazioni di fair value intervenute successivamente all'avvio della copertura contabile, purché non superi il suo valore recuperabile. Le variazioni del fair value dello strumento di copertura e dell'elemento coperto sono rilevate nella sezione D) del conto economico.

Qualora la variazione del valore del fair value dell'elemento coperto sia maggiore in valore assoluto della variazione del fair value dello strumento di copertura, la differenza tra le due variazioni di fair value è rilevata nella voce di conto economico interessata dall'elemento coperto e non nell'area D (quindi, ad esempio, in caso di copertura del magazzino, tale effetto andrà nelle voci riferite alla variazione del magazzino) [9].

\subsubsection{Copertura dei flussi finanziari (cash flow hedge)}

In un'operazione di copertura dei flussi finanziari l'obiettivo strategico della direzione aziendale è di norma quello di stabilizzare i flussi finanziari attesi di un elemento coperto quale, ad esempio, l'interesse variabile pagato periodicamente su 
un debito finanziario, un impegno all'acquisto o vendita di beni, oppure un'operazione programmata altamente probabile dalla quale scaturirà per esempio un acquisto o una vendita di beni.

Nel modello contabile della copertura dei flussi finanziari, ad ogni chiusura di bilancio, la società rileva nello stato patrimoniale lo strumento di copertura al fair value e in contropartita alimenta la voce A) VII "Riserva per operazioni di copertura dei flussi finanziari attesi". Tale riserva di patrimonio netto non può accogliere le componenti inefficaci della copertura contabile, ossia variazioni di fair value dello strumento finanziario derivato alle quali non corrisponde una variazione di segno contrario dei flussi finanziari attesi dell'elemento coperto. Qualora, infatti, l'ammontare delle variazioni di fair value intervenute nello strumento di copertura sia superiore all'ammontare in valore assoluto delle variazioni di valore intervenute sull'elemento coperto dall'inizio della relazione di copertura, l'eccedenza rappresenta la parte di inefficacia della copertura. La componente di inefficacia è rilevata nella sezione D) del conto economico.

Il rilascio della riserva per operazioni di copertura dei flussi finanziari attesi deve avvenire come segue:

a) in una copertura di flussi finanziari connessi ad un'attività o passività iscritta in bilancio o di un'operazione programmata altamente probabile o impegno irrevocabile l'importo della riserva deve essere riclassificato a conto economico nello stesso esercizio o negli stessi esercizi in cui i flussi finanziari futuri coperti hanno un effetto sull'utile (o sulla perdita) d'esercizio (per esempio, negli esercizi in cui sono rilevati gli interessi attivi o gli interessi passivi o quando si verifica l'operazione programmata). La voce di conto economico in cui classificare il rilascio della riserva è la stessa che è impattata dai flussi finanziari attesi quando hanno effetto sull'utile (o sulla perdita) d'esercizio;

b) in una copertura dei flussi finanziari connessi ad un'operazione programmata altamente probabile o impegno irrevocabile che comportano successivamente la rilevazione di un'attività o passività non finanziarie, la società al momento della rilevazione dell'attività o della passività deve eliminare l'importo dalla riserva per operazioni di copertura dei flussi finanziari attesi e includerlo direttamente nel valore contabile dell'attività (nei limiti del valore recuperabile) o della passività;

Tuttavia, in presenza di una riserva negativa se la società non prevede di recuperare tutta la perdita o parte della riserva in un esercizio o in più esercizi futuri, la società deve immediatamente imputare alla voce D) 19) d) del conto economico dell'esercizio la riserva o la parte di riserva che non prevede di recuperare.

\subsection{I derivati di copertura e la relazione di copertura}

La contabilizzazione delle operazioni di copertura sopradescritta è applicabile alle operazioni di copertura se, e soltanto se, tutte le seguenti condizioni sono soddisfatte:

a) la relazione di copertura consiste solo di strumenti di copertura ammissibili ed elementi coperti ammissibili (si rimanda rispettivamente ai successivi paragrafi 5.3.1 e 5.3.2 per l'illustrazione di quali siano in dettaglio tali strumenti di copertura ed elementi coperti ammissibili);

b) ai sensi dell'articolo 2426 comma 1, numero 11 bis), del Codice Civile "si considera sussistente la copertura in presenza, di stretta e documentata correlazione tra le caratteristiche dello strumento o dell'operazione coperti e quelle dello strumento di copertura". Pertanto all'inizio della relazione di copertura vi deve essere una designazione e una documentazione formale della relazione di copertura, degli obiettivi della società nella gestione del rischio e della strategia nell'effettuare la copertura. La documentazione deve includere l'individuazione dello strumento di copertura, dell'elemento coperto, della natura del rischio coperto e di come la società valuterà se la relazione di copertura soddisfi i requisiti di efficacia della copertura (compresa la sua analisi delle fonti di inefficacia della copertura e di come essa determina il rapporto di copertura);

c) la relazione di copertura soddisfa tutti i seguenti requisiti di efficacia della copertura:

i. vi è una relazione economica tra l'elemento coperto e lo strumento di copertura. Ciò implica che il valore dello strumento di copertura varia al variare, in relazione al rischio oggetto della copertura, nella direzione opposta di quello dell'elemento coperto. Ci si deve pertanto attendere una variazione sistematica del valore dello strumento di copertura e del valore dell'elemento coperto in conseguenza ai movimenti della stessa variabile sottostante. La verifica di tale relazione economica può avvenire in termini sia qualitativi, sia quantitativi (si rimanda a tal proposito al successivo paragrafo 5.4);

ii. l'effetto del rischio di credito della controparte dello strumento finanziario derivato e dell'elemento coperto, qualora il rischio di credito non sia il rischio oggetto di copertura, non prevale sulle variazioni di valore risultanti dalla relazione economica. Pertanto ci si attende che il rischio di credito non incida significativamente sul fair value dello strumento di copertura e dell'elemento coperto;

iii. viene determinato il rapporto di copertura pari al rapporto tra le quantità di strumenti finanziari derivati utilizzati e le quantità di elementi coperti. Normalmente questo rapporto è 1:1 (uno strumento finanziario derivato copre esattamente l'elemento coperto) sebbene in alcuni casi possa essere differente. Il calcolo del rapporto di copertura deve essere tale da non determinare ex ante l'inefficacia della copertura (esempio copertura di un nozionale superiore di quello dell'elemento coperto).

\subsubsection{Strumenti di copertura ammissibili}

Il principio stabilisce che qualsiasi derivato può essere designato come strumento di copertura, ad eccezione di un'opzione (put o call) venduta, o una combinazione di derivati che abbia lo stesso effetto di un'opzione venduta. In tal caso il derivato 
non è ammissibile come strumento di copertura a meno che sia designata a compensazione di un'opzione (put o call) acquistata.

Inoltre, il derivato deve essere designato come strumento di copertura nella sua interezza. Non è infatti consentito suddividere il fair value in componenti e designare una o più di queste come strumento di copertura.

Ad eccezione della regola generale di designazione dell'intero derivato quale copertura, possono essere designati come strumenti di copertura di flussi finanziari:

a) il solo valore intrinseco (o variazione di valore del prezzo a pronti) del fair value di un'opzione (put o call) acquistata. Il valore intrinseco dell'opzione rappresenta la differenza tra il prezzo di mercato unitario del sottostante e il prezzo di esercizio dell'opzione moltiplicata per il sottostante. Ciò che residua rispetto al fair value dell'opzione è il valore temporale, ossia la probabilità che, con il passare del tempo, il valore intrinseco aumenti;

b) il solo elemento spot di un contratto forward. Similmente a quanto previsto per le opzioni, anche per i contratti forward è possibile designare come strumento di copertura esclusivamente il valore intrinseco (nel caso del forward il cosiddetto spot element);

c) una quota dell'intero strumento di copertura, quale ad esempio il $50 \%$ del sottostante. La restante quota rappresenta uno strumento finanziario derivato non di copertura. Tuttavia, lo strumento di copertura non può essere designato per una parte del periodo di tempo in cui lo strumento è in circolazione.

\subsubsection{Elementi coperti ammissibili}

Sono ammissibili come elementi coperti, sia singolarmente che raggruppati, i seguenti elementi o loro componenti:

a) attività e passività iscritte in bilancio;

b) impegni irrevocabili;

c) operazioni programmate altamente probabili.

Un elemento di patrimonio netto non può essere designato come elemento coperto. Può essere oggetto di copertura anche un'esposizione aggregata, ossia una combinazione tra un elemento coperto e uno strumento finanziario derivato.

Se l'elemento coperto è un'operazione programmata (o sua componente), il verificarsi in futuro di tale operazione deve essere altamente probabile. Ciò significa che la probabilità di verificarsi dell'operazione deve essere ben superiore al 51\%. L'OIC 32 dedica buona parte dell'appendice $\mathrm{C}$ del principio contabile in commento per spiegare secondo quali criteri debba avvenire la stima della probabilità di accadimento di un'operazione programmata, chiarendo così il concetto di "altamente probabile".

\subsection{La verifica della relazione di copertura}

\subsubsection{Verifica della relazione di copertura in via qualitativa}

È frequente riscontrare che le operazioni di copertura sono poste in essere mediante strumenti finanziari derivati aventi caratteristiche del tutto simili a quelle dell'elemento coperto, quali la scadenza, il valore nominale, la data di regolamento dei flussi finanziari ed il sottostante (definite "relazioni di copertura semplici") e in cui il fair value dello strumento finanziario derivato è prossimo allo zero alla data di rilevazione iniziale. In tali casi alla data di rilevazione iniziale la loro contabilizzazione può essere basata su un'analisi esclusivamente qualitativa e non necessita dell'elaborazione di un test quantitativo.

L'OIC 32 chiarisce infatti che la verifica della relazione economica può avvenire in via qualitativa quando gli elementi portanti dello strumento di copertura e dell'elemento coperto corrispondono o sono strettamente allineati. Sono "elementi portanti": l'importo nominale; la data di regolamento dei flussi finanziari; la scadenza e la variabile sottostante. In tal caso è possibile concludere che il valore dello strumento di copertura evolve nella direzione opposta di quello dell'elemento coperto per effetto di uno stesso rischio e che, quindi, tra elemento coperto e strumento di copertura esiste una relazione economica non casuale. In ogni caso è necessario verificare la sussistenza del requisito di cui al punto c) ii) del precedente paragrafo 5.3, ossia che l'effetto del rischio di credito non incida significativamente sul fair value dello strumento di copertura e dell'elemento coperto.

Nella newsletter di maggio 2017 l'OIC ha fornito, a seguito della ricezione di una richiesta, un chiarimento riguardo la possibilità di applicare il modello contabile ad hoc dello OIC 32, previsto per le relazioni di copertura semplici, a tutte quelle operazioni di copertura di flussi finanziari laddove sussista piena identità tra elemento coperto e strumento di copertura eccettuata la scadenza [10]. La fattispecie sottoposta all'OIC era quella di una società che decide di accendere un finanziamento a tasso variabile di lungo termine e di coprirsi dal rischio di tasso mediante la stipula di un contratto finanziario derivato. Il richiedente sottolineava come lo strumento di copertura fosse stipulato con i medesimi "elementi portanti" del correlato elemento coperto, ad eccezione della scadenza (ad esempio 20 anni per il finanziamento e 5 anni per il derivato). L'OIC ha chiarito in tale sede che in casi come questo, laddove le scadenze dei flussi di cassa designati come elemento coperto coincidano, o siano strettamente allineate, a quelle dello strumento di copertura e siano soddisfatti tutti gli altri criteri previsti dai punti a), b) e c) del paragrafo 5.3, il modello semplificato può comunque essere attivato.

\subsubsection{Verifica della relazione di copertura in via quantitativa}

Per una valutazione sotto il profilo quantitativo della relazione economica il principio dispone che sia possibile ricorrere a varie metodologie, anche statistiche, normalmente utilizzate in azienda nell'attività di risk management. Infatti, l'esistenza di 
una correlazione statistica fra due variabili rappresenta un'utile indicazione che potrebbe esistere una relazione economica efficace tra lo strumento di copertura e l'elemento coperto. Per correlazione statistica si intende una relazione tra due variabili statistiche tale che a ciascun valore della prima variabile corrisponda con una "certa regolarità" un valore della seconda. Non si tratta necessariamente di un rapporto di causa-effetto, ma semplicemente della tendenza di una variabile a variare in funzione di un'altra. Talvolta le variazioni di una variabile dipendono dalle variazioni dell'altra, talvolta sono comuni e talvolta sono reciprocamente dipendenti.

In alcune circostanze, specialmente in presenza di variabili sottostanti differenti tra elemento coperto e strumento di copertura, può essere necessario corroborare la mera analisi statistica con altre valutazioni quantitative. È questo il caso, ad esempio, delle tecniche utilizzate per la determinazione del livello di inefficacia attraverso la tecnica del derivato ipotetico o altre metodologie quali, ad esempio, studi di sensitività. La valutazione in via quantitativa della relazione economica può essere utilizzata per il calcolo della componente di inefficacia della copertura da determinare per le coperture dei flussi finanziari e da rilevare nella sezione $\mathrm{D})$ del conto economico.

Inoltre, la valutazione in via quantitativa deve essere sempre effettuata in sede di valutazione successiva dei criteri di ammissibilità per la contabilizzazione delle operazioni di copertura. Infatti, la verifica della sussistenza dei criteri di ammissibilità deve essere fatta in via continuativa. Ad ogni data di chiusura del bilancio la società deve valutare se la relazione di copertura soddisfi ancora i requisiti di efficacia e, in caso di cambiamenti significativi nella relazione economica tra elemento coperto e strumento di copertura oppure di incremento significativo del rischio di credito, è necessario applicare le regole dettate dal principio contabile in tema di revisione o di cessazione di una relazione di copertura.

Nella seguente Figura 6 si illustrano graficamente i passaggi previsti dall'OIC 32 per attivare la copertura contabile.

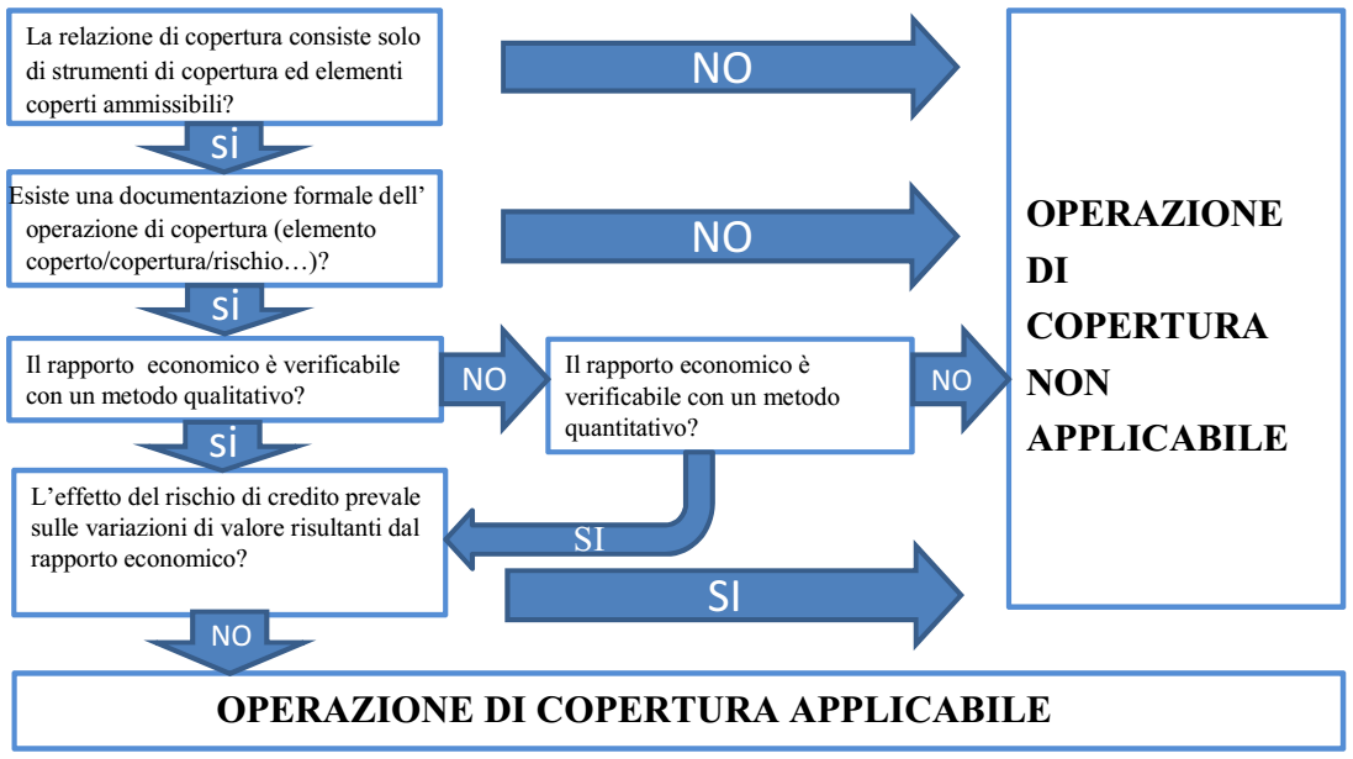

Figura 6. Diagramma di flusso per attivazione della copertura ai sensi dell'OIC 32 [11]

\section{Conclusioni}

Il lavoro ha riguardato i criteri di contabilizzazione dei derivati alla luce della normativa attualmente in vigore in tema di redazione del bilancio d'esercizio. Come si è avuto modo di vedere, le novità recentemente introdotte apportano un significativo contributo per migliorare la disclosure tecnico contabile dei contratti derivati sottoscritti da imprese di tipo nonfinancial che redigono il bilancio secondo i principi contabili nazionali.

Dai dati di mercato attualmente disponibili, emerge che le imprese di tipo non-financial utilizzano i contratti derivati tipicamente per coprirsi dal rischio valuta e dal rischio tasso di interesse. Dal confronto dei dati diffusi da Banca d'Italia relativamente alle posizioni in derivati over-the-counter a fine dicembre 2017 e fine dicembre 2016, emerge che nell'ultimo anno è aumentato l'ammontare delle posizioni in derivati in cui la controparte è un'istituzione non finanziaria, specialmente per la copertura del rischio tasso d'interesse.

Il recepimento della direttiva 2013/34/UE ha comportato un'importante innovazione nella prassi contabile nazionale, introducendo l'obbligo per tutte le imprese, incluse anche le imprese che redigono il bilancio in forma abbreviata ai sensi dell'articolo 2435 bis del Codice Civile, di valutazione al fair value degli strumenti finanziari derivati.

L'Italia ha recepito nel proprio ordinamento interno la nuova direttiva contabile con l'entrata in vigore del D.Lgs n. 139/2015 (Decreto Bilanci), il quale introduce per la prima volta con decorrenza $1^{\circ}$ gennaio 2016 l'obbligo di rilevazione in bilancio degli strumenti finanziari derivati.

A fronte dell'entrata in vigore delle nuove norme civilistiche, l'Organismo Italiano di Contabilità ha emanato il principio contabile OIC 32, il quale disciplina i criteri di rilevazione, classificazione e valutazione degli strumenti finanziari derivati detenuti dalle imprese per finalità speculative o di copertura. Il principio contabile de quo pone particolare attenzione sulla 
contabilizzazione dei derivati nelle operazioni di copertura, prevedendo un regime differenziato a seconda che la copertura si riferisca al fair value di elementi presenti nel bilancio (fair value hedge) oppure a flussi finanziari o operazioni di futura manifestazione (cash flow hedge).

Ai fini della corretta valutazione in bilancio dei derivati di copertura è dunque importante verificare la relazione di copertura, ossia la relazione economica tra strumento di copertura ed elemento coperto. Per eseguire tale verifica il principio contabile OIC 32 prevede due metodi alternativi: uno semplificato di tipo qualitativo ed uno quantitativo. In alcune circostanze, l'impossibilità di applicare il modello contabile semplificato, implica l'obbligo di dimostrare quantitativamente l'efficacia della relazione di copertura. Ciò può comportare la necessità per le imprese di sviluppare nuove competenze e dotarsi degli strumenti tipici di risk management necessari per verificare la relazione copertura di un derivato, anche in via quantitativa.

\section{Mattia Fabbri e Pier Giuseppe Giribone}

\section{Bibliografia}

[1] Imad A. Moosa, Exchange rate forecasting: techniques and applications, Palgrave Macmillan, 9 (2000)

[2] Gordon M. Bodnar, Costanza Consolandi, Giampaolo Gabbi, Ameeta Jaiswal-Dale, A Survey on risk Management and Usage of Derivatives by Non-Financial Italian Firms, Carefin, Università Bocconi, Research Paper No. 7/08 (2008)

[3] Mariano Graziano, Le imprese italiane e gli strumenti derivati, Banca d'Italia, 7-15 (2012)

[4] Triennial Central Bank Survey, BIS Monetary and Economic Department, 3-8 (2016)

[5] Comunicato stampa Banca d'Italia, Rilevazione sui prodotti derivati over-the-counter a dicembre 2016 (2017)

[6] Comunicato stampa Banca d'Italia, Rilevazione sui prodotti derivati over-the-counter a dicembre 2017 (2018)

[7] Fabrizio Bava, Derivati di copertura: le scritture contabili degli IRS sugli interessi di mutui", in Il Fisco 16/2016 Contabilità e Bilancio, Wolters Kluwer, 1560 (2016)

[8] Relazione illustrativa allo Schema di decreto legislativo recante attuazione della direttiva 2013/34/EU, 9 (2015)

[9] Alberto Quagli, Bilancio e Principi Contabili, Wolters Kluwer, 294 e 307-308 (2017)

[10] Organismo Italiano di Contabilità, Emendamenti ai Principi Contabili Nazionali, 23-26 (2017)

[11] Organismo Italiano di Contabilità, OIC 32 Strumenti Finanziari Derivati, Appendice D, 46 (2016) 


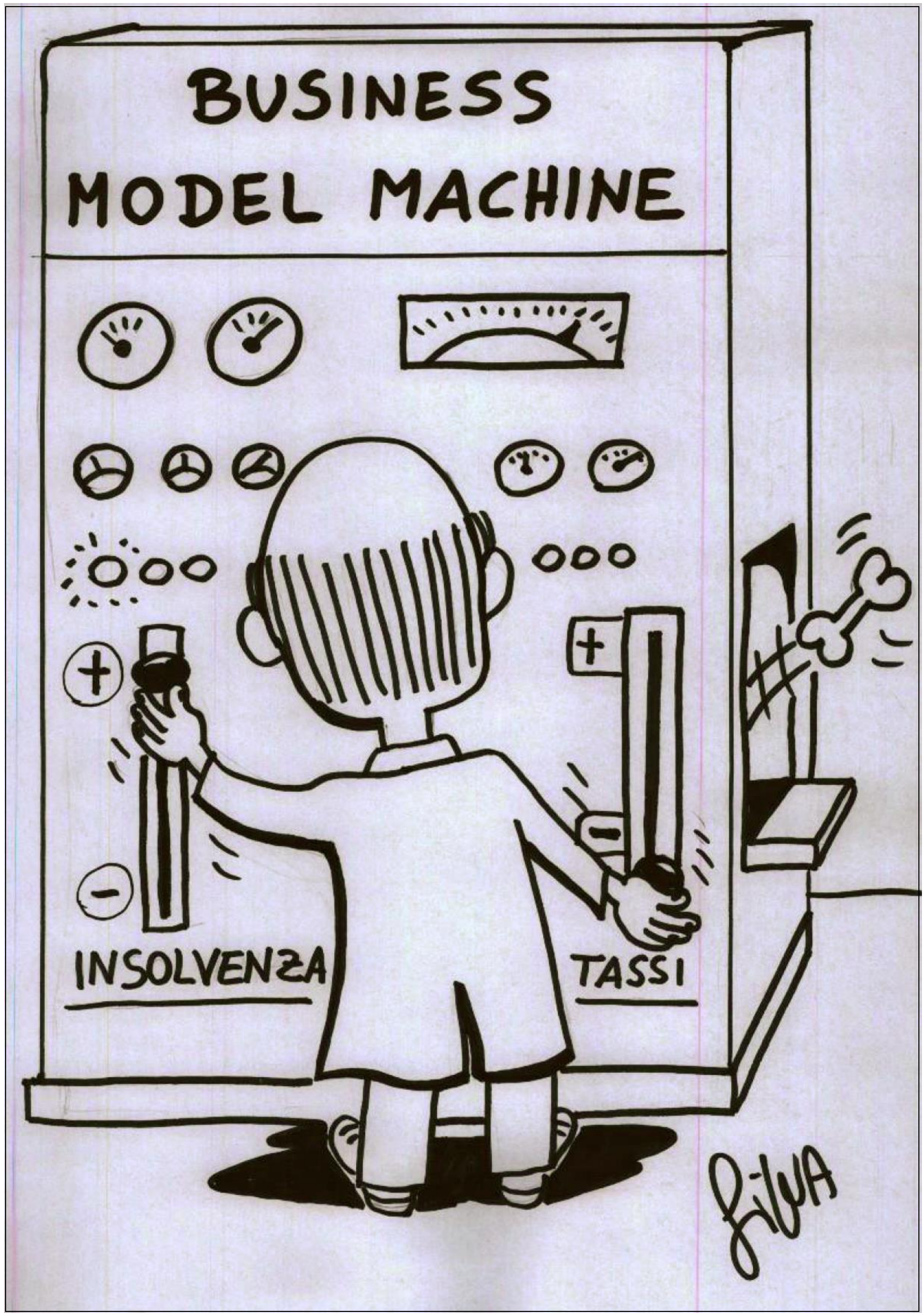

RISK MANAGEMENT MAGAZINE ANNO 13 N$^{\circ} 1$ - PAGINA - 38 - 
Risk Management Magazine

Anno 13, n 1 Gennaio - Aprile 2018

Direttore Responsabile

Maurizio Vallino

Condirettore

Corrado Meglio

Consiglio Scientifico

Giampaolo Gabbi (Direttore del Consiglio Scientifico),

Ruggero Bertelli,

Paola Bongini,

Anna Bottasso,

Marina Brogi

Ottavio Caligaris,

Simona Cosma,

Paola Ferretti,

Andrea Giacomelli,

Adele Grassi,

Pier Giuseppe Giribone,

Duccio Martelli,

Laura Nieri

Anna Grazia Quaranta,

Pasqualina Porretta

Enzo Scannella,

Cristiana Schena,

Giuseppe Torluccio

Comitato di redazione

Sara Brianza

Emanuele Diquattro

Fausto Galmarini

Rossano Giuppa

Aldo Letizia

Paolo Palliola

Enzo Rocca

Fabio Salis

Vignettista: Silvano Gaggero

Proprietà, Redazione e Segreteria:

Associazione Italiana Financial Industry Risk Managers (AIFIRM), Via Sile 18, 20139 Milano

Registrazione del Tribunale di Milano $\mathrm{n}^{\circ} 629$ del 10/9/2004

E-mail: segreteria@ aifirm.it; Tel. 3896946315 Lunedì - Venerdì h.15-17

Stampa: Algraphy S.n.c. - Passo Ponte Carrega 62-62r, 16141 Genova

Le opinioni espresse negli articoli impegnano unicamente la responsabilità dei rispettivi autori

SPEDIZIONE IN ABBONAMENTO POSTALE AI SOCI AIFIRM RESIDENTI IN ITALIA, IN REGOLA CON L'ISCRIZIONE 


\section{RISK MANAGEMENT MAGAZINE}

Rivista dell'Associazione Italiana Financial Industry Risk Managers

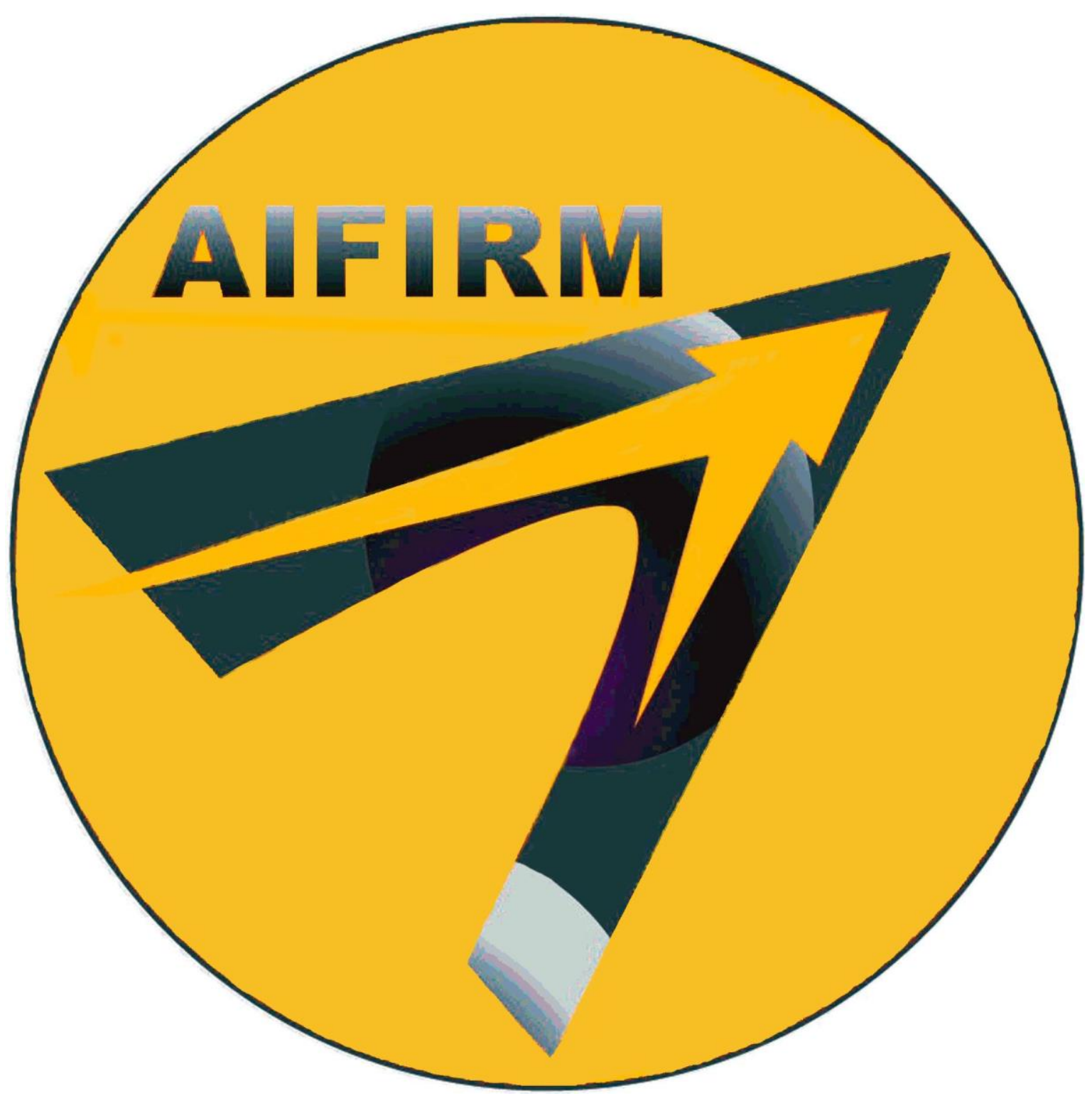

